\title{
Vortex shedding effects in grid-generated turbulence
}

\author{
G.Melina*1, P. J. K. Bruce $^{1}$, and J. C. Vassilicos ${ }^{1}$ \\ ${ }^{1}$ Department of Aeronautics, Imperial College London, London SW7 2AZ, UK
}

May 17, 2016

\begin{abstract}
The flow on the centerline of grid-generated turbulence is characterised via hot-wire anemometry for 3 grids with different geometry: a regular grid (RG60), a fractal grid (FSG17) and a single square grid (SSG). Thanks to a higher value of the thickness $t_{0}$ of its bars, SSG produces greater values of turbulence intensity $T u$ than FSG17, despite SSG having a smaller blockage ratio. However the higher Tu for SSG is mainly due to a more pronounced vortex shedding contribution. The effects of vortex shedding suppression along the streamwise direction $x$ are studied by testing a new 3D configuration, formed by SSG and a set of four splitter plates detached from the grid $(\mathrm{SSG}+\mathrm{SP})$. When vortex shedding is damped, the centerline location of the peak of turbulence intensity $x_{\text {peak }}$ moves downstream and $T u$ considerably decreases in the production region. For FSG17 the vortex shedding is less intense and it disappears more quickly, in terms of $x / x_{\text {peak }}$, when compared to all the other configurations. When vortex shedding is attenuated, the integral length scale $L_{u}$ grows more slowly in the streamwise direction, this being verified both for FSG17 and for SSG+SP. In the production region, there is a correlation between the vortex shedding energy and the skewness and the flatness of the velocity fluctuations. When vortex shedding is not significant, the skewness is highly negative and the flatness is much larger than 3. On the opposite side, when vortex shedding is prominent, the non-Gaussian behaviour of the velocity fluctuations becomes masked.
\end{abstract}

\section{Introduction}

During the last decade multiscale/fractal-generated turbulence has been widely investigated, both experimentally (e.g. Hurst and Vassilicos, 2007; Valente and Vassilicos, 2011; Nagata et al., 2013; Weitemeyer et al., 2013; Goh et al., 2014; Gomes-Fernandes et al., 2015; Nedić et al., 2015; Cafiero et al., 2015; Baj et al., 2015) and with Direct Numerical Simulations (DNS) (e.g. Nagata et al., 2008; Laizet and Vassilicos, 2012; Suzuki et al., 2013; Zhou et al., 2014; Laizet and Vassilicos, 2015; Dairay et al., 2015). At a large distance from a perturbing grid in its turbulence decay region on the centreline, where the turbulence intensity $T u$ decreases along the streamwise direction $x$, fractal square grids with blockage ratio $\sigma=25 \%$ produce higher values of $T u$ if compared to a regular grid with $\sigma=34 \%$ and with a similar effective mesh size $M_{\text {eff }}$ for the same inlet velocity $U_{\infty}$ and for the same dimensional distance $x$ from the grid (Mazellier and Vassilicos, 2010). Laizet and Vassilicos (2015) performed DNS simulations of the flow downstream of fractal grids and of regular grids with comparable $\sigma$ and similar $M_{\text {eff }}$ and for a similar Reynolds number based on $M_{\text {eff }}$. The latter study shows that when averaging the turbulence intensity over a plane parallel to the grid, for the same $x$ this is higher for fractal grids than for regular grids downstream of the location of its peak value. However the same study shows that upstream of the location of this maximum, the plane-averaged turbulence intensity is higher for regular grids than for fractal grids. The distance $x_{\text {peak }}$ from the grid, where $T u$ is maximum on the centerline, is the streamwise extent of the turbulence production region, where $T u$ increases with $x$, and is representative of the location where the wakes, originating from the largest bars of the grid, meet on the centreline. The distance $x_{\text {peak }}$ can be approximately predicted in terms of the wake-interaction length scale $x^{*}=L_{0}^{2} / t_{0}$ (Mazellier and Vassilicos, 2010), where $L_{0}$ is

*Email address for correspondence: g.melina13@imperial.ac.uk 
the length of the bars of the largest iteration of the square pattern, and $t_{0}$ is their thickness in a plane normal to the direction of the flow.

Gomes-Fernandes et al. (2012) theoretically motivated and experimentally demonstrated that (i) $x_{\text {peak }} / x^{*}$ is inversely proportional to the drag coefficient $c_{d}$ of the largest bars of the grid and that (ii) the value of $T u$ at $x_{\text {peak }}, T u_{\text {peak }}$, is proportional to $c_{d} t_{0} / L_{0}$. The latter result suggests that we can use a grid made of a single square (Laizet et al., 2015) designed with a large ratio $t_{0} / L_{0}$, so that $T u$ is high while $\sigma$, and presumably also the static pressure drop, is small.

Fractal geometries have proved to be an effective solution for suppressing vortex shedding downstream of particular objects. In axisymmetric turbulent wakes produced by fractal plates, the vortex shedding energy is reduced by up to $60 \%$ compared to the case of circular and square plates with the same frontal area (Nedić et al., 2015). Nedić and Vassilicos (2015) showed that by increasing the number of fractal iterations in an airfoil's (NACA 0012) trailing edge with multiscale modifications, the energy of vortex shedding decreases too. It is believed that the fractal modification of the perimeter affects the vortex shedding formation mechanism and re-distributes the turbulent kinetic energy among a broader range of scales (frequencies).

Mazellier and Vassilicos (2010) discovered that, downstream of fractal square grids, strong rare decelerating flow events occur in the turbulence production region. As a result, the probability density functions (PDFs) of the velocity fluctuations $u$ appear highly left-skewed and characterised by large values of flatness. On the contrary, advancing further downstream in the turbulence decay region, the skewness and the flatness of $u$ gradually get close to values typical of a Gaussian distribution. These observations lead to some new research questions. (1) Are these features typical of fractal grids or are they also observable with regular and single-scale grids? (2) Which phenomena can affect the magnitude of these events, given in particular that in the turbulence production region the wakes shed from the largest bars of the grid have not fully met yet. (3) Does vortex shedding play a significant role in the production region, especially when the value of $t_{0}$ is considerably high? (4) If yes, how are the statistics of the velocity fluctuations affected by the vortex shedding energy content? (5) Is vortex shedding attenuated in the production region of fractal grids, as a result of the presence of the smaller geometrical iterations?

The aim of this paper is to provide some answers to these research questions. We first characterise the flow downstream of three types of turbulence-generating grids placed in a wind tunnel. We consider a regular grid (RG60), a fractal square grid (FSG17) and a single square grid (SSG) with the highest value of $t_{0} / L_{0}$. We perform single-component hot-wire measurements downstream of the grids, mainly on the centerline. We also quantify the static pressure drop along the centerline by traversing a Pitot-static tube. We focus on some of the effects induced by the vortex shedding originating from the largest bars of the grids. For this purpose we also consider a novel 3D turbulence generator (SSG+SP) which is formed by SSG and a set of four splitter plates detached from it.

It is well known that, among passive techniques, vortex shedding suppression by using splitter plates is one of the simplest and most effective solutions (Akilli et al., 2005). Roshko (1954) showed that, when a long splitter plate is attached downstream of a circular cylinder with diameter $D, c_{d}$ is reduced as a result of the vortex shedding suppression. Apelt and West (1975) performed experiments on splitter plates past bluff bodies and investigated the effect of $L_{s p}$, where $L_{s p}$ is the length of the splitter plate. They found that regular vortex shedding is completely suppressed when the reattachment of the flow occurs on the splitter plate. This happens for $L_{s p} / D \geq 3$ for a plate normal to the flow and for $L_{s p} / D \geq 5$ for a circular cylinder. This last result also holds for a splitter plate attached to a rectangular prism, as shown by Ali et al. (2011). However, when long splitter plates are used, a well-developed vortex street arises from the combined bluff body/splitter plate. Vortex shedding can also be reduced using a shorter detached splitter plate placed at a distance $x_{s p}$ from the bluff body, where $x_{s p}$ is measured until the splitter plate's leading edge. Roshko (1954) found that by using a splitter plate with $L_{s p} / D=1.14$ detached from a circular cylinder at $R e_{D}=14500$, an optimal position exists for which vortex shedding attenuation is maximum and $c_{d}$ is minimum; $R e_{D}$ is the Reynolds number based on $D$. This optimum occurs for $x_{s p} / D=2.7$. Similar results were obtained by performing DNS at lower $R e_{D}$. Lin and $\mathrm{Wu}$ (1994) found an optimal distance $x_{s p} / D=2.5$ for $L_{s p} / D=2$ and $R e_{D}=100$, Hwang et al. (2003) 


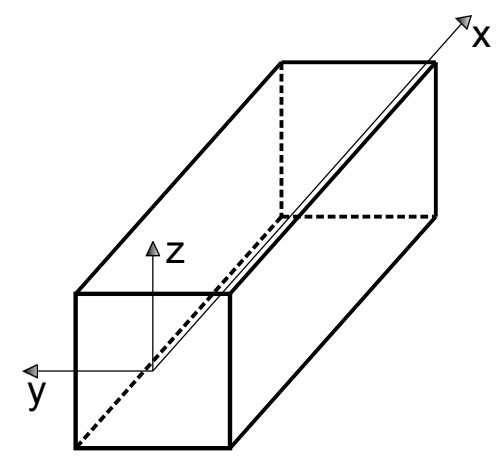

Figure 1: Sketch of the wind tunnel's test section.

report an optimal value $x_{s p} / D=2.7$ for $L_{s p} / D=1$ and $R e_{D}=160$.

In this work we identify an optimal distance $x_{s p} / t_{0}$ between the splitter plates and SSG from a limited number of such distances that we have been able to experiment with. We estimate the energy associated with a band of frequencies centred on the vortex shedding frequency (vortex shedding energy) for the configuration with and without splitter plates. We quantify the vortex shedding energy content for FSG17 and we compare it to SSG and SSG+SP at the same values of $x / x_{\text {peak }}$ along the centerline. We highlight the effects of the vortex shedding attenuation. In particular we study how the vortex shedding energy affects the skewness and the flatness of the velocity fluctuations in the turbulence production region.

The remainder of this paper is structured as follows: in section 2 we describe the experimental technique and the data reduction process; in section 3 we characterise the turbulent flow for RG60, FSG17 and SSG; in section 4 we discuss the effects of vortex shedding suppression along the centerline; finally section 5 concludes.

\section{Experimental details}

\subsection{Wind tunnel}

Experiments have been performed in a low speed open-loop wind tunnel. Its maximum velocity, when empty, is $33 \mathrm{~m} / \mathrm{s}$ with a background turbulence intensity of $0.1 \%$. The working section is $3 \mathrm{~m}$ long with a square cross section $T^{2}=0.46^{2} \mathrm{~m}^{2}$. A sketch of the wind tunnel's test section is shown in figure 1 in order to define the spatial coordinate notation used in this paper. The inlet velocity $U_{\infty}$ upstream of the grid is imposed by measuring the static pressure difference across the wind tunnel's contraction with a micromanometer Furness Control FCO510. In the present measurements $5 \mathrm{~m} / \mathrm{s} \leq U_{\infty} \leq 17 \mathrm{~m} / \mathrm{s}$. The boundary layer displacement thickness in the empty wind tunnel (no grids at the inlet) is estimated to be lower than $10 \mathrm{~mm}$ at $x=3 \mathrm{~m}$ for the minimum $U_{\infty}$, $U_{\infty}=5 \mathrm{~m} / \mathrm{s}$. The temperature of the flow is measured with a thermocouple placed at the inlet of the test section, the ambient pressure is measured with an absolute pressure gauge connected to the micromanometer.

\subsection{Turbulence-generating grids}

In this work, three different turbulence-generating grids are placed at the inlet $(x=0)$ of the wind tunnel's test section. The grids extend over the entire size of the cross section of the wind tunnel. Scaled diagrams of the grids are illustrated in figure 2. The first grid is a regular bi-planar grid (RG60) which has a blockage ratio $\sigma=32 \%$. The second grid is a multiscale fractal square grid (FSG17) which has been widely studied and documented in several previous experiments (see Hurst and Vassilicos, 2007; Seoud and Vassilicos, 2007; Mazellier and Vassilicos, 2010; Valente and Vassilicos, 2011, 2014; Laizet et al., 2015). This grid has four iterations $(N=4)$ and $\sigma=25 \%$. The thickness ratio is $t_{r}=t_{0} / t_{N-1}=17$, where $t_{N-1}$ is the thickness of the smallest bars. The ratio of the lengths of the bars of two successive iterations is $R_{L}=L_{j+1} / L_{j}=0.5$, the ratio of their thickness is $R_{t}=t_{j+1} / t_{j}=t_{r}^{1 /(1-N)}=0.39(j=0,1,2)$. Finally, the third grid is a single square grid 




(a) RG60

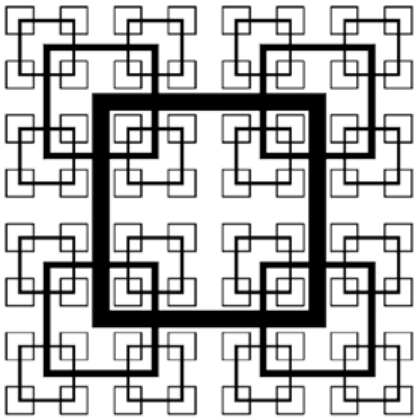

(b) FSG17

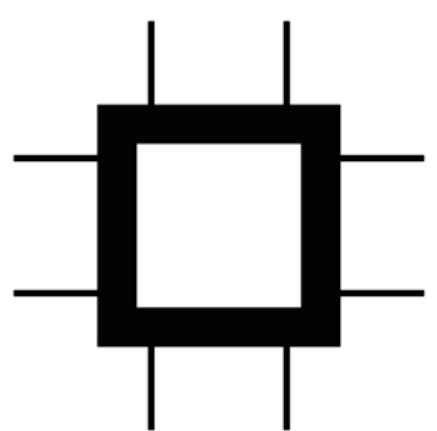

(c) SSG

Figure 2: Turbulence-generating grids used in the experiments.

\begin{tabular}{cccccccc} 
Grid & $L_{0}[\mathrm{~mm}]$ & $t_{0}[\mathrm{~mm}]$ & $x^{*}[\mathrm{~mm}]$ & $c_{0} / t_{0}$ & $c_{d}$ & $\sigma[\%]$ & $R_{t_{0}}$ \\
\hline RG60 & 60.0 & 10.0 & 360.0 & 1.00 & 2.14 & 32 & $3310-11260$ \\
FSG17 & 237.8 & 19.2 & 2945.3 & 0.32 & 2.25 & 25 & $6360-21620$ \\
SSG & 229.0 & 43.0 & 1219.6 & 0.13 & 2.01 & 20 & $14240-48410$
\end{tabular}

Table 1: Geometrical parameters of the grids and inlet Reynolds numbers $R e_{t_{0}}$ for $U_{\infty}=5-17 \mathrm{~m} / \mathrm{s}$.

(SSG) with $\sigma=20 \%$. This is a single-scale grid and it is simply made of one thick square supported by eight thin struts (6 mm thick). The SSG is designed to obtain high values of $T u$ while keeping low values of $\sigma$, i.e. increasing the ratio $t_{0} / L_{0}$, but still allowing a sufficiently high value of $x^{*}$ in order to generate an extended turbulence production region.

The geometrical details of the grids and the inlet Reynolds numbers based on $t_{0}, R e_{t_{0}}=U_{\infty} t_{0} / \nu$, are summarised in table $1 ; \nu$ is the kinematic viscosity of air. We report the sectional drag coefficients $c_{d}$ of the largest bars of the grids, which are estimated by interpolating the experimental values collected in Munshi et al. (1999) as a function of the aspect ratio $c_{0} / t_{0}$, where $c_{0}$ is the depth of the bars in the $x$ direction (chord).

\subsection{Splitter plates}

The primary goal of this paper is to assess the importance of vortex shedding in the production region of grid-generated turbulence. Given that SSG is the grid with the largest value of $t_{0}$, we expect that the effect of vortex shedding will be most pronounced in its production region. For this reason we design a static device to be placed downstream of SSG in such a way that the vortex shedding mechanism is attenuated, allowing an assessment of the grid behaviour with different levels of intensity of vortex shedding.

This is a set of four splitter plates that, when connected together, form an open box where the distance between two parallel plates matches the value of $L_{0}$, therefore every element is aligned along the median line of the bars of SSG (see figure 3). The length of the splitter plates in the $x$ direction is $L_{s p}=64.5 \mathrm{~mm}$, so that $L_{s p} / t_{0}=1.5$. The thickness of the plates is $t_{s p}=5 \mathrm{~mm}$, the ratio $t_{s p} / t_{0}$ is 0.116 . For a circular cylinder with diameter $D$ at $R e_{D}=5500$, Akilli et al. (2005) did not find appreciable differences in their experimental results using splitter plates with three different thickness ratios $\left(t_{s p} / D=0.016,0.04,0.08\right)$. The turbulence intensity downstream of the splitter plates (without the presence of grids at the inlet of the wind tunnel) does not exceed $0.25 \%$ for $0<x<2.3 \mathrm{~m}$ on the centreline.

The plates are connected to an outer square frame thanks to eight supporting struts aligned with those supporting SSG. The distance $x_{s p}$ between the leading edge of the splitter plates and the grid can be freely varied. Preliminary measurements were made with values of $x_{s p} / t_{0}$ between 0 and 5 in order to identify an optimal distance at which the splitter plates are most effective. In this paper we henceforth refer to the configuration formed by SSG and the set of splitter plates as SSG+SP (see figure 3). 


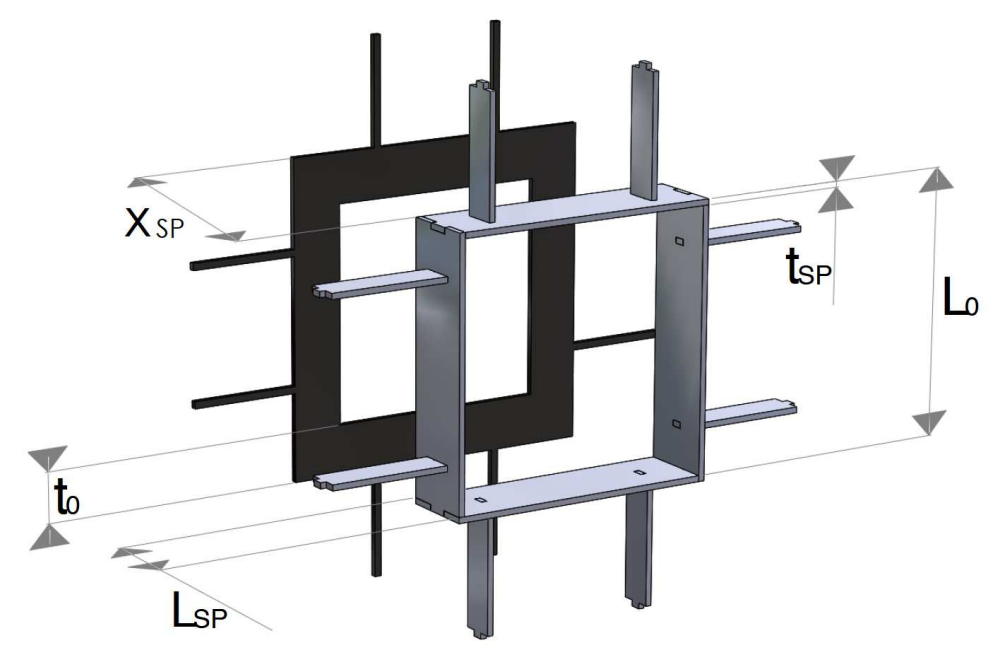

Figure 3: Sketch of SSG with splitter plates (SSG+SP).

\subsection{Velocity and pressure measurements}

Velocity measurements have been performed via single-component hot-wire anemometry. The hot-wire is made from a Wollaston wire with a $5 \mu \mathrm{m}$ diameter $\left(d_{w}\right)$ platinum core which is soft-soldered on a Dantec 55P01 hot-wire probe. The wire sensing element is obtained by etching its central part with a nitric acid bath. The resulting sensing length is about $1 \mathrm{~mm}$ long $\left(l_{w}\right)$, thus giving an aspect ratio $l_{w} / d_{w}$ of about 200 . The hot-wire probe is mounted on a Dantec 55H21 support coupled to a traverse system which allows movement along the $x$ and the $z$ directions. The hot-wire probe is operated by a Dantec Streamline Pro constant temperature anemometer system. The hot wire is systematically calibrated before and after each experimental run against the free-stream velocity of the wind tunnel. The calibrations are obtained using fourth-order polynomial fits of the velocity as a function of the voltage at constant temperature. The conditioned signal is sampled at $100 \mathrm{kHz}$, with the analogue low-pass filter on the Streamline set at $30 \mathrm{kHz}$, using a National Instruments-6229 data acquisition system connected to a computer. The hot-wire measurements are performed at $U_{\infty}=5 \mathrm{~m} / \mathrm{s}$ and $17 \mathrm{~m} / \mathrm{s}$ for all the grids. Moreover, for the comparison between SSG and SSG+SP, measurements are also performed at an intermediate velocity, $U_{\infty}=11 \mathrm{~m} / \mathrm{s}$. In order to obtain converged statistics, the sampling time for each measurement point on the centerline is set to $300 \mathrm{~s}$, which corresponds to at least $29000-97000$ integral time scales for the minimum and maximum $U_{\infty}$ respectively. The sampling time for the measurements relative to the vertical velocity profiles (along $z$ ) is reduced to 120 s since in this case we are only interested in low-order statistics (mean velocity and turbulence intensity).

The Reynolds decomposition of the instantaneous velocity signal $\tilde{u}(t)$ is $\tilde{u}(t)=U+u(t)$, where $U$ is the time-averaged value, $U=\langle\tilde{u}(t)\rangle$, and $u(t)$ is the fluctuating component. The turbulence intensity $T u$ is computed with $T u=u^{\prime} / U$, where $u^{\prime}$ is the root-mean-square value of $u(t), u^{\prime}=\sqrt{\left\langle u(t)^{2}\right\rangle}$. The longitudinal integral time scale $\Theta$ is calculated from the power spectrum density $E_{u}(f)$ of $u(t)$ in the frequency domain $f$ as (Pope, 2000):

$$
\Theta=\frac{E_{u}(0)}{4 u^{\prime 2}}
$$

The longitudinal integral length scale (correlation length scale) $L_{u}$ is obtained from $\Theta$ by applying Taylor hypothesis, $L_{u}=\Theta U$. As previously done in Seoud and Vassilicos (2007) and Mazellier and Vassilicos (2010), the kinetic energy dissipation rate per unit mass $\varepsilon$ is estimated from:

$$
\varepsilon=15 \nu<\left(\frac{\partial u}{\partial x}\right)^{2}>
$$


where:

$$
<\left(\frac{\partial u}{\partial x}\right)^{2}>=\int_{0}^{+\infty} k^{2} E_{u}(k) d k
$$

under the assumption of isotropy for the small scales, which is shown to hold by the results of DNS of turbulent flows downstream of similar grids (Laizet et al., 2015). Frequencies $f$ and $E_{u}(f)$ are converted to wavenumbers $k$ and $E_{u}(k)$ by means of Taylor hypothesis, $k=2 \pi f / U, E_{u}(k)=U E_{u}(f) /(2 \pi)$. The Taylor microscale $\lambda$ is evaluated using its isotropic definition, $\lambda^{2}=15 \nu u^{\prime 2} / \varepsilon$, the Kolmogorov length scale $\eta$ is computed from $\eta=\left(\nu^{3} / \varepsilon\right)^{1 / 4}$, finally the dissipation coefficient $C_{\varepsilon}$ is evaluated from $C_{\varepsilon}=\varepsilon L_{u} / u^{\prime 3}$.

For the hot-wire measurements performed at $U_{\infty}=5 \mathrm{~m} / \mathrm{s}$, the frequency response of the hot-wire is high enough to resolve the dissipation spectrum $k^{2} E_{u}(k)$ up to $k \eta=1$ and above. For the measurements relative to SSG and SSG+SP at $U_{\infty}=11 \mathrm{~m} / \mathrm{s}$, the maximum resolvable $k \eta$ is at worst $k \eta \approx 0.75$, therefore the dissipation is in this case slightly underestimated (up to $2 \%$ ). When considering instead the measurements performed at $U_{\infty}=17 \mathrm{~m} / \mathrm{s}$, the resolution is considerably reduced up to $k \eta \approx 0.3$ for RG60, $k \eta \approx 0.65$ for FSG17, $k \eta \approx 0.5$ for SSG and $k \eta \approx 0.6$ for SSG+SP at worst. Taking as a reference the measurements at our lowest free-stream velocity, we estimate that for $U_{\infty}=17 \mathrm{~m} / \mathrm{s}$ the values of $\varepsilon$ would be underestimated by up to $22 \%$ for RG60, $3 \%$ for FSG17, $7 \%$ for SSG and $4 \%$ for SSG+SP. Given the figures above, in the present paper we do not consider any results derived from $\varepsilon$ (such as $\lambda$ and $C_{\varepsilon}$ ) for the measurements performed at $U_{\infty}=17 \mathrm{~m} / \mathrm{s}$.

Static pressure measurements are performed along the centerline by traversing a straight Pitot-static tube downstream of the grids for $U_{\infty}=5-17 \mathrm{~m} / \mathrm{s}$. The difference between the local static pressure $p$ (from the Pitot-static port) and the free-stream static pressure $p_{\infty}$ (from pressure taps located upstream of the grids) is acquired using a second micromanometer. The pressure drop coefficient $C_{\Delta p}$ is evaluated from:

$$
C_{\Delta p}=\frac{p-p_{\infty}}{\frac{1}{2} \rho U_{\infty}^{2}}
$$

where $\rho$ is the density of air at the ambient pressure and at the fluid temperature.

\section{Flow downstream of the grids}

\subsection{Basic flow documentation}

Figure 4 shows the normalised mean velocity $U / U_{\infty}$ and the turbulence intensity $T u$ along the centerline downstream of RG60, FSG17 and SSG. For all three cases the mean velocity is a maximum close to the grid and successively decreases towards $U_{\infty}$ proceeding further downstream. For RG60 the mean velocity is found to increase slightly with $x$ for $x>0.6 \mathrm{~m}$, owing to the growth of a turbulent boundary layer on the wind tunnel walls (see appendix A). For FSG17 the mean velocity remains considerably higher than $U_{\infty}$ (about $10 \%$ ) even far from the grid if compared to both RG60 and SSG. This observation for FSG17 is in good agreement with the values previously reported by Mazellier and Vassilicos (2010). For SSG the normalised mean velocity is found to be slightly lower for $U_{\infty}=5 \mathrm{~m} / \mathrm{s}$ in the interval $0<x<1 \mathrm{~m}$. However, advancing further downstream, the values of $U / U_{\infty}$ collapse with the results relative to $U_{\infty}=17 \mathrm{~m} / \mathrm{s}$.

For RG60 the turbulence intensity is high in the region close to the grid, reaching a peak value $T u_{\text {peak }}$ of about $18 \%$ at $x_{\text {peak }}=0.11 \mathrm{~m}$, before rapidly dropping with downstream distance to below $5 \%$ for $x>0.9 \mathrm{~m}$. For FSG17 and SSG the value of $T u_{\text {peak }}$ is lower, about 9\% for FSG17 and 15\% for SSG, and it occurs for a larger distance from the grid, $x_{\text {peak }}=1.18 \mathrm{~m}$ for FSG17 and $x_{\text {peak }}=0.61 \mathrm{~m}$ for SSG. The latter feature is a direct result of the greater values of the wake-interaction length scale for FSG17 and SSG. The position $x_{\text {peak }}$ can be expressed as $x_{\text {peak }}=k_{1} c_{d}^{-1} x^{*}$, where $k_{1}$ is a factor depending on the free-stream (upstream of the grids) turbulence intensity and on the geometry of the grid (Gomes-Fernandes et al., 2012). The factor $k_{1}$ is close to 0.9 for a laminar free-stream (as is the case for the present measurements) and fractal grids with $\sigma=25 \%$ and with $t_{r}=8,13,17$ (Gomes-Fernandes et al., 2012). In our measurements we find $x_{\text {peak }} / x^{*}=0.3,0.4,0.5$ for RG60, FSG17 and SSG respectively, therefore the corresponding values of $k_{1}$ are $0.64,0.9$ and 1 . The difference 


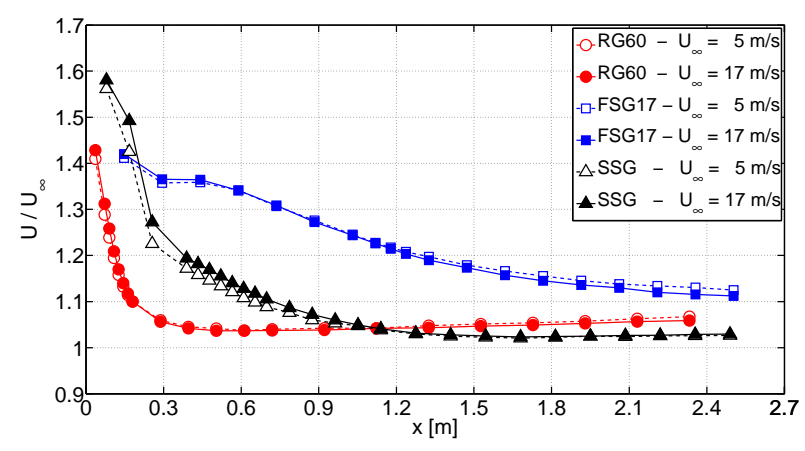

(a)

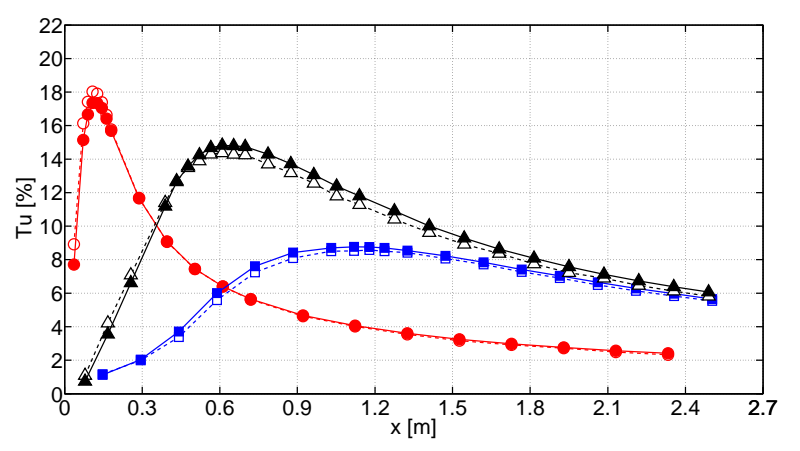

(b)

Figure 4: Mean velocity (a) and turbulence intensity (b) for RG60, FSG17 and SSG along the centerline.

in the values of $k_{1}$ between FSG17 and SSG can be attributed to the difference between the blockage ratios of the two grids (Laizet and Vassilicos, 2011). Laizet et al. (2015) have experimental evidence where $x_{\text {peak }} / x^{*}$ is a decreasing function of $\sigma$. The value of $k_{1}$ is found to be significantly lower for RG60. This difference might be due both to the higher blockage ratio of RG60 and also to the fact that, differently from FSG17 and SSG, this grid is regular and bi-planar. This is in agreement with the observations made in Gomes-Fernandes et al. (2012), who found the value of $k_{1}$ for the fractal grids used in their experiment to be higher than that for the regular square-mesh grids of Cardesa-Dueñas et al. (2012).

In the turbulence production region and close to $x_{\text {peak }}$ the values of $T u$ for SSG, which has the lowest $\sigma$, are considerably higher if compared to FSG17. The reason for this is related to the higher ratio $t_{0} / L_{0}$ for SSG which is more than double that for FSG17. Note that the values of turbulence intensity for a fractal square grid are higher than those for a single square grid with the same $t_{0} / L_{0}$ (Zhou et al., 2014). The physical argument which can explain the nature of the higher turbulence intensity in the production region of our SSG is further discussed in section 4. In the turbulence decay region, the values of Tu for FSG17 approach those for SSG, both being considerably higher than those for RG60.

Figure 5 shows vertical profiles of $U$ and $T u$ (normalised by their value on the centerline) for $0 \leq z / L_{0} \leq 0.5$, at a series of streamwise locations in the turbulence decay regions of the grids. It is clear that the mean velocity profiles become more homogeneous as one moves downstream and that RG60 seems to reach the best level of homogeneity when compared to the other grids, with its profiles becoming completely flat at $x \geq 0.72 \mathrm{~m}$. However one must consider that $x^{*}$ (and so $x_{\text {peak }}$ ) is substantially smaller for RG60. Therefore if we made a comparison at the same $x$, we would not be taking into account that the flow is much further away from its production region for this grid than for FSG17 and SSG. In fact the position $x=0.72 \mathrm{~m}$ corresponds to $x / x_{\text {peak }}=6.67$ for RG60, which is a value that we never reach for either FSG17 or SSG in the present wind tunnel. A similar observation can be made when comparing the homogeneity of the mean velocity between FSG17 and SSG. If we compare the profiles for FSG17 at $x=2.21 \mathrm{~m}$ (or $x=2.50 \mathrm{~m}$ ) with the ones for SSG at $x=2.22 \mathrm{~m}$ ( or $x=2.49 \mathrm{~m}$ ), we conclude that the mean velocity downstream of SSG is more homogeneous. On the contrary when we compare the profiles measured at the same $x / x_{\text {peak }}$, for example $x / x_{\text {peak }}=1.87$ for both FSG17 and SSG, we instead conclude that FSG17 and SSG exhibit the same level of homogeneity since $U_{z / L_{0}=0.5} / U_{z / L_{0}=0}$ is about 0.93 for FSG17 and 0.91 for SSG.

When comparing the vertical profiles of $T u$ in figure 5 we notice that FSG17 differs from the other two grids. For both RG60 and SSG the turbulence intensity is a monotonically increasing function of $z$ for $0 \leq z / L_{0} \leq 0.5$. On the contrary for FSG17 the profiles of $T u$ exhibit a local maximum around $z / L_{0}=0.25$. This feature is most probably due to the presence of the smaller geometrical iterations on the fractal square grid. We observe that $z / L_{0}=0.25$ is the coordinate where the profiles of the mean velocity for FSG17 and RG60 show an inflection point, i.e. $\left(\partial^{2} U / \partial z^{2}\right)_{z / L_{0}=0.25}=0$. If we now reasonably assume that $|\partial U / \partial z|>>|\partial W / \partial x|$ (and $|\partial U / \partial y|>>|\partial V / \partial x|)$, for FSG17 the position where $T u$ is maximum corresponds to the location where the absolute value of the $y$-component (or alternatively of the $z$-component for symmetry considerations) of the 


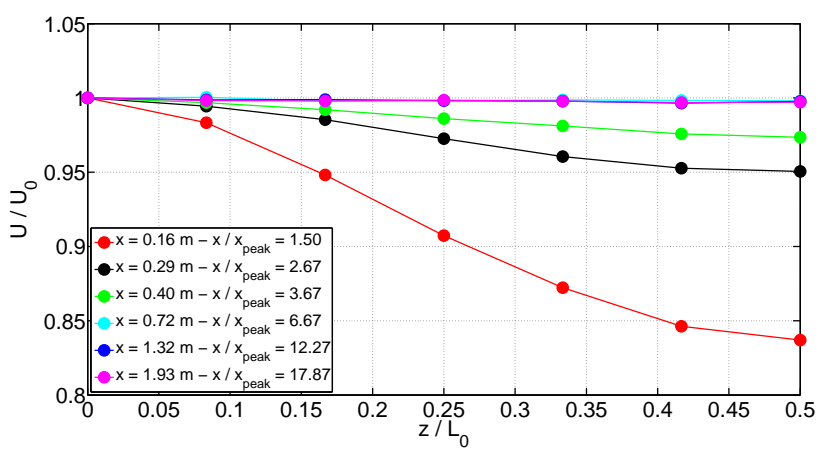

(a) RG60

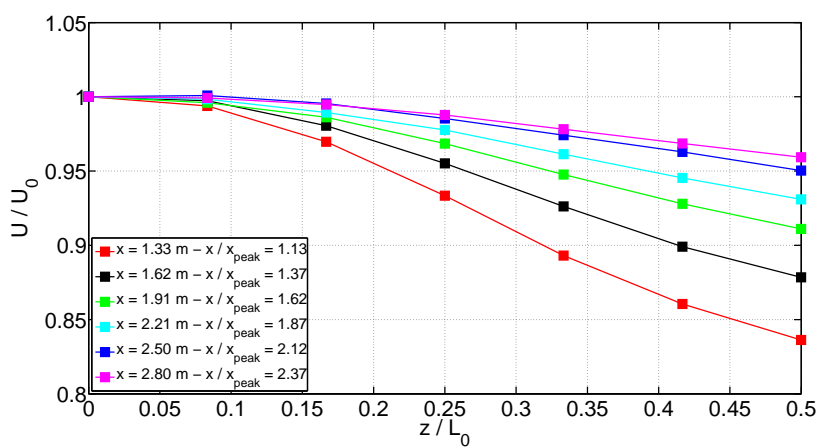

(c) FSG17

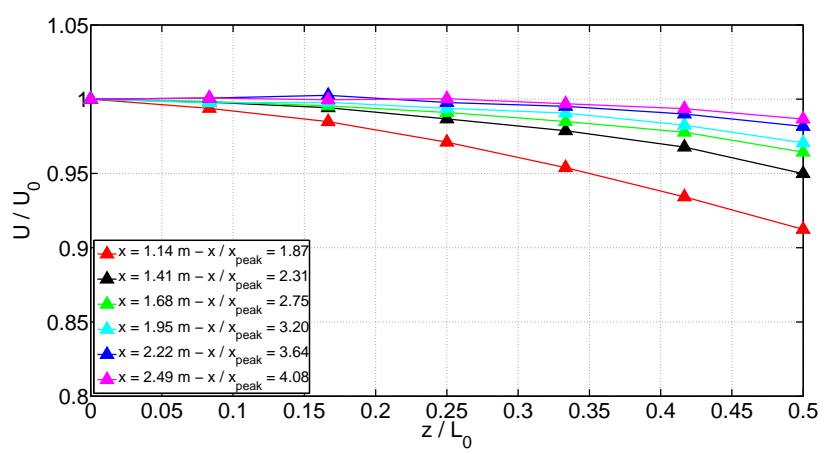

(e) SSG

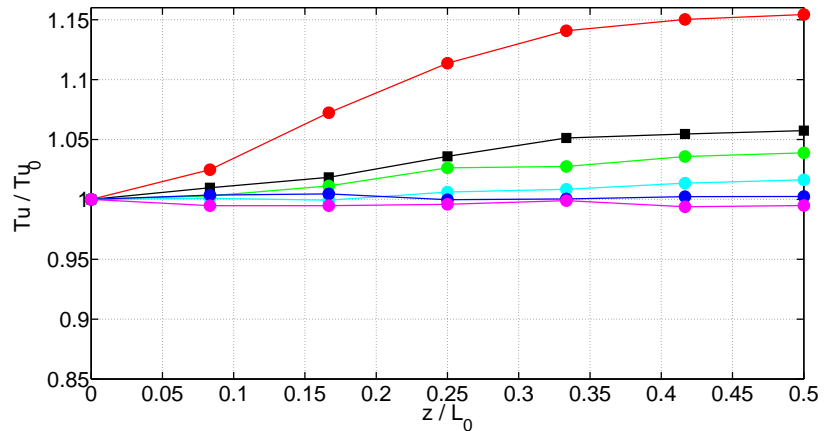

(b) RG60

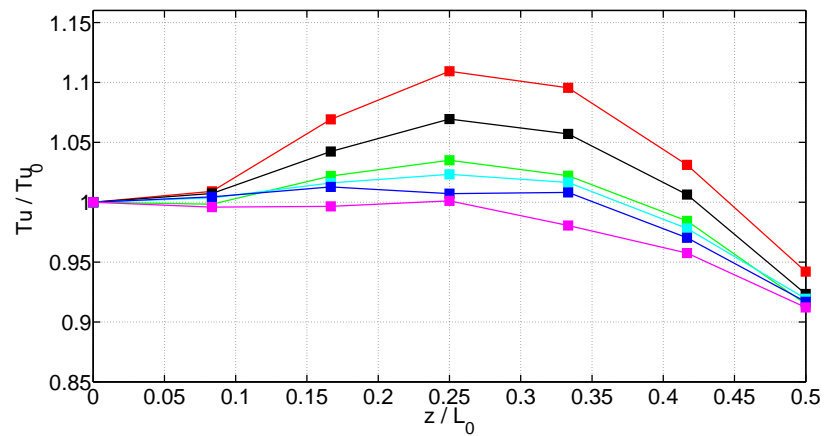

(d) FSG17

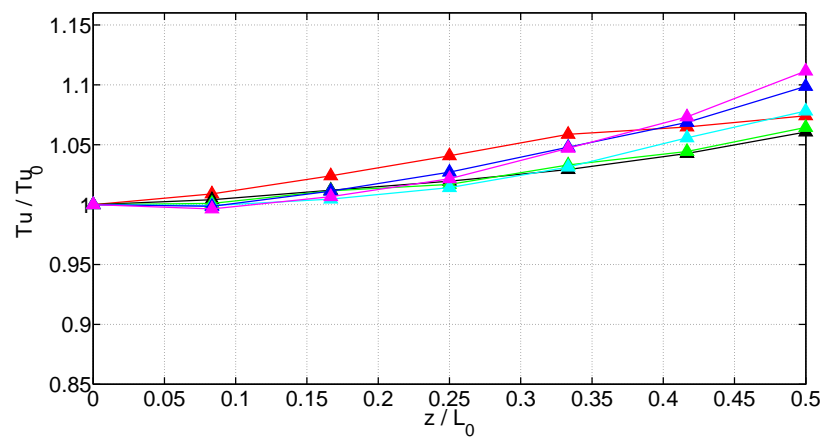

(f) SSG

Figure 5: Vertical profiles of mean velocity (left) and turbulence intensity (right) for RG60 (top), FSG17 (center) and SSG (bottom). $U_{0}$ and $T u_{0}$ are respectively the mean velocity and the turbulence intensity on the centerline; $U_{\infty}=17 \mathrm{~m} / \mathrm{s}$.

mean vorticity, $\partial U / \partial z-\partial W / \partial x \approx \partial U / \partial z(\partial V / \partial x-\partial U / \partial y \approx-\partial U / \partial y)$, is also maximum.

The pressure drop coefficient $C_{\Delta p}$ (equation 4) along the centerline is plotted against $x$ and $x / x_{\text {peak }}$ in figure 6 for the three grids. The absolute value of $C_{\Delta p}$ in the far decay region is maximum for RG60 whereas it is minimum for SSG, consistently with the decreasing blockage ratio of the grids. On the other hand the pressure recovery length, that is the distance after which the pressure should remain constant, is the highest for SSG. We have to remark that in our case the pressure drop coefficient does not reach a well-defined constant value but it is found to slightly decrease along $x$. This can be explained by the growth of the boundary layer on the walls of the wind tunnel (the mean velocity increases and the static pressure decreases). We notice that for SSG the values of $C_{\Delta p}$ are notably higher for $U_{\infty}=5 \mathrm{~m} / \mathrm{s}$ than for $U_{\infty}=17 \mathrm{~m} / \mathrm{s}$ in the interval $0<x<1 \mathrm{~m}$ $\left(0<x / x_{\text {peak }}<1.65\right)$, which coincides with the region where we have observed a lower mean velocity (see figure 4 a).

Figure 7a shows the evolution of the longitudinal integral length scale $L_{u}$ along the centerline for the different grids, where $L_{u}$ has been normalised with the tunnel width $T$. For each of the three grids there is a satisfactory 


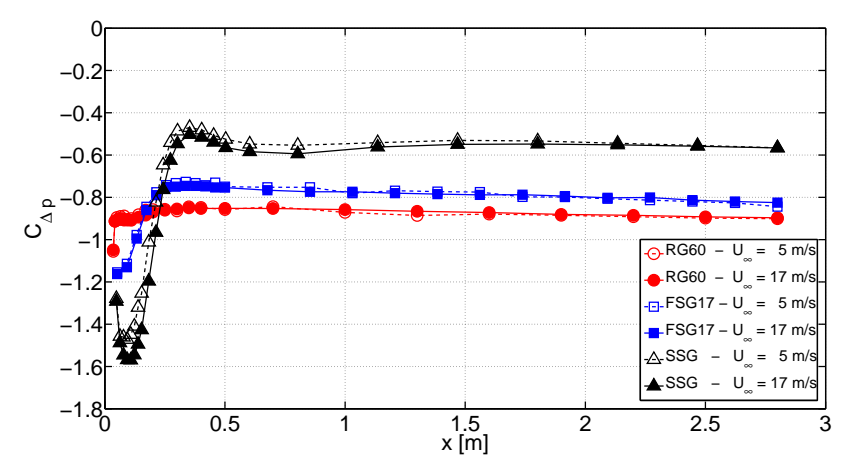

(a)

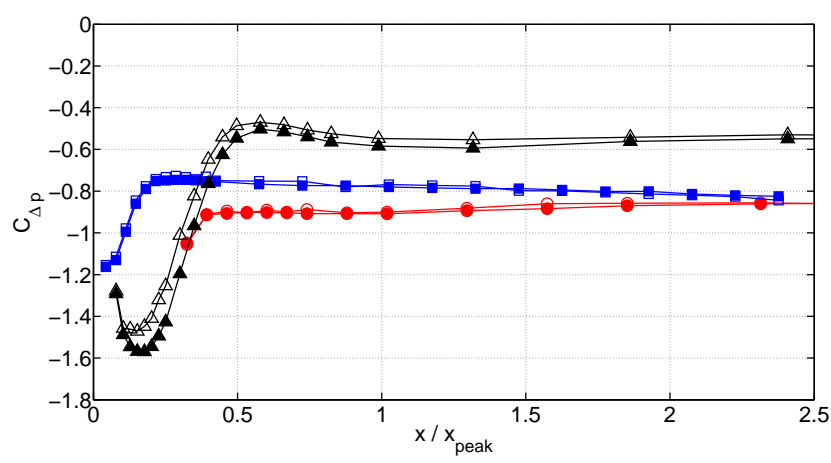

(b)

Figure 6: Pressure drop coefficient as a function of $x$ (a) and of $x / x_{\text {peak }}$ (b) for RG60, FSG17 and SSG along the centerline.

collapse of the measurements taken at two different free-stream velocities, indicating that $L_{u}$ is invariant with $U_{\infty}$, at least for the range investigated in this study. This means that the downstream evolution of this length scale is set by the geometry of the grids only and not by the inlet Reynolds number. For FSG17 and SSG, the very first measurement positions in the turbulence production region are characterized by large values of $L_{u}$ since the flow is still intermittent there ( $L_{u}$ is much larger in a laminar than in a turbulent flow). Advancing further downstream in the turbulence decay regions, the values of $L_{u}$ are considerably lower for RG60 due to the fact that $L_{0}$ is smaller for RG60 than for FSG17 and SSG. These two grids have very similar $L_{0}$ and consequently comparable values of $L_{u}$. However FSG17 exhibits the slowest growth of $L_{u}$ with $x$, similarly to what was originally observed by Hurst and Vassilicos (2007). It is interesting to look at the ratio between the integral and the Taylor length scales $L_{u} / \lambda$ (figure $7 \mathrm{~b}$ ), which gives an indication of the separation between the large and the small scales of the turbulent fluctuations. In the region where $C_{\varepsilon}$ is constant for fixed inlet conditions, the ratio $L_{u} / \lambda$ should decrease in proportion to $R e_{\lambda}$, where $R e_{\lambda}=u^{\prime} \lambda / \nu$. Comparison of the plots in Figures $7 \mathrm{~b}$ and $7 \mathrm{c}$ shows that this feature holds for RG60 when $x>0.6 \mathrm{~m}\left(x / x_{\text {peak }}>5.5\right.$ ), where $C_{\varepsilon}$ (figure 7d) approaches a constant value. On the contrary for FSG17, as already previously discussed in Seoud and Vassilicos (2007), in the turbulence decay region the ratio $L_{u} / \lambda$ remains approximately constant, despite $R e_{\lambda}$ clearly decreasing. Here we show that the same feature can be also observed for SSG. This indicates that, for both FSG17 and SSG, our measurements are always performed in the non-equilibrium region of turbulence where $C_{\varepsilon} \neq$ constant. In this region $C_{\varepsilon} \propto R e_{\lambda}^{-1}$, which implies $L_{u} / \lambda=$ constant for fixed inlet conditions.

\subsection{Production region}

We make a comparison between two streamwise positions on the centerline, one in the production region $\left(x / x_{\text {peak }}<1\right)$ and the other in the decay region $\left(x / x_{\text {peak }}>1\right)$. The positions have been chosen to give similar values of $x / x_{\text {peak }}$ for each grid: $x / x_{\text {peak }}=0.67,0.62,0.64$ in the production region and $x / x_{\text {peak }}=1.67,1.75,1.72$ in the decay region for RG60, FSG17 and SSG respectively.

In figure 8 (a, c, e) we show the power spectrum density $E_{u}$ for the measurements taken at $U_{\infty}=5 \mathrm{~m} / \mathrm{s}$. Frequencies have been converted to Strouhal numbers $S t$ using a reference frequency given by $U_{\infty} / t_{0}$, and the energy density has been normalised with $\Theta u^{\prime 2}$. For each spectrum the cut-off frequency is chosen to be $f_{c u t}=1.2 f_{\eta}$, where $f_{\eta}$ is the Kolmogorov frequency, $f_{\eta}=U /(2 \pi \eta)$. In the inertial range all the spectra exhibit a Kolmogorov-like power law decay, $E_{u} \sim S t^{-p}$ with $p$ close to $5 / 3$, both in the production region and in the decay region. For all the grids, in the production region the inertial range is observable for higher frequencies and it is more extended than for the decay region, in agreement with the recent observations made in Laizet et al. (2015). In addition to this, the extent of the inertial range is larger for FSG17 and SSG than for RG60.

In the production region the spectra corresponding to RG60 and SSG show a clear peak which is due to the presence of vortex shedding from the bars of the grids. The intensity of this peak gets attenuated as one 


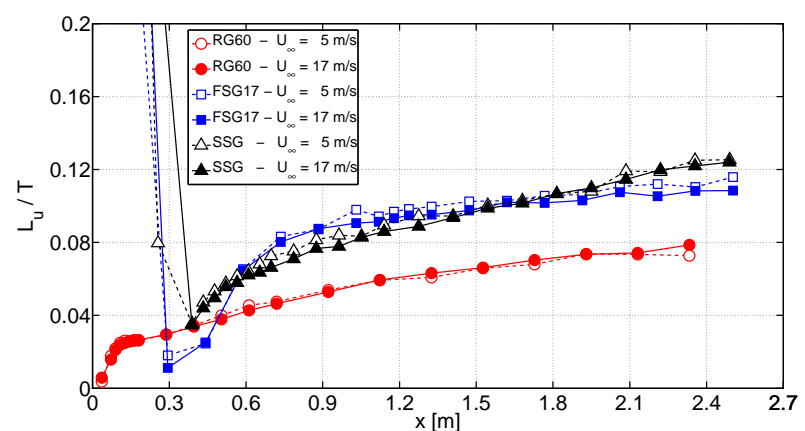

(a)

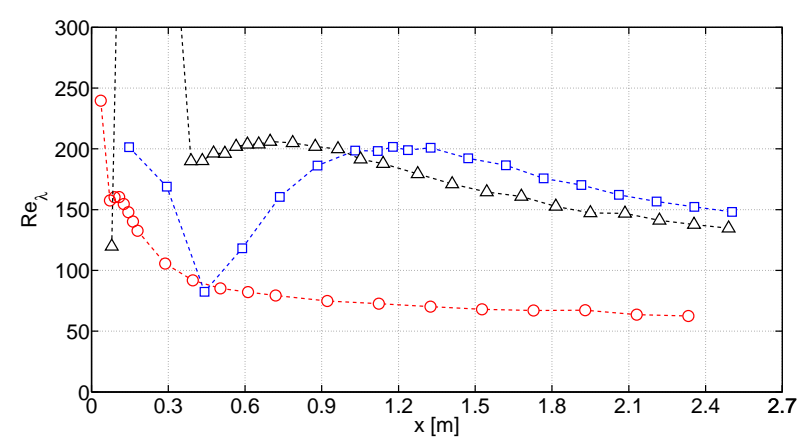

(c)

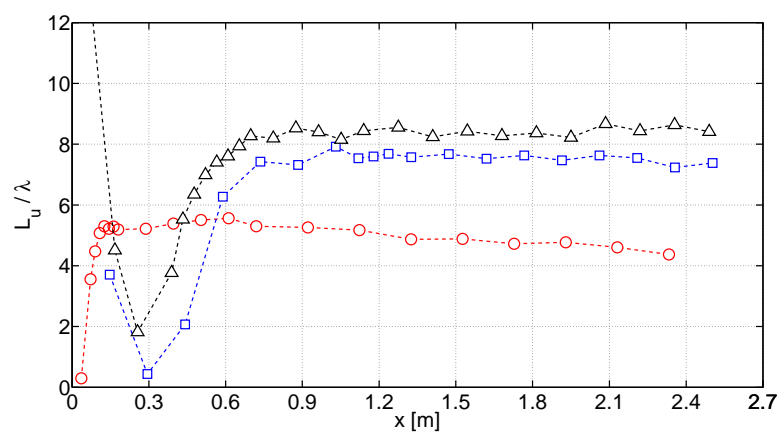

(b)

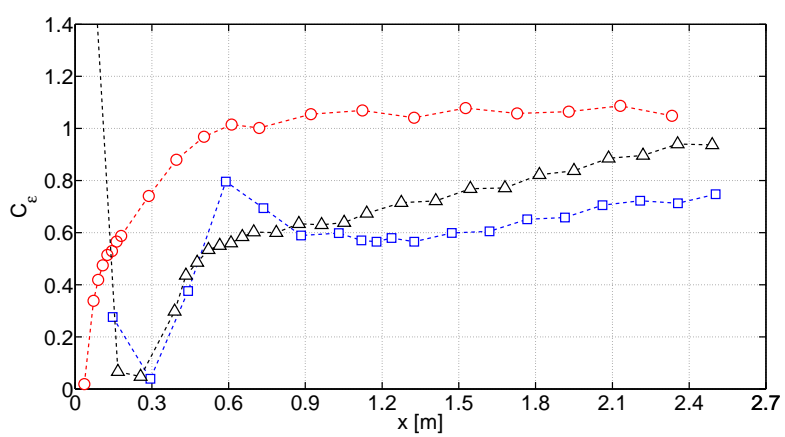

$(\mathrm{d})$

Figure 7: Integral length scale (a), ratio between integral and Taylor length scales (b), Reynolds number based on Taylor length scale (c) and dissipation coefficient (d) for RG60, FSG17 and SSG along the centerline. Data for $U_{\infty}=17 \mathrm{~m} / \mathrm{s}$ are not shown for quantities derived from $\varepsilon$ as explained in section 2.4 .

\begin{tabular}{c|c|c|c|c} 
& \multicolumn{2}{|c|}{$S t_{s h}$} & \multicolumn{2}{c}{$S t_{s h}^{A}$} \\
$U_{\infty}[\mathrm{m} / \mathrm{s}]$ & 5 & 17 & 5 & 17 \\
\hline RG60 & 0.163 & 0.167 & 0.399 & 0.409 \\
FSG17 & 0.125 & 0.126 & 0.440 & 0.443 \\
SSG & 0.187 & 0.189 & 0.432 & 0.436
\end{tabular}

Table 2: Vortex shedding Strouhal numbers $S t_{s h}$ and $S t_{s h}^{A}$ for RG60, FSG17 and SSG; $U_{\infty}=5 \mathrm{~m} / \mathrm{s}$ and $17 \mathrm{~m} / \mathrm{s}$.

proceeds downstream in the decay region but, at the considered locations, it is still detectable for both RG60 and SSG. If we now consider the case of FSG17, in the production region the vortex shedding phenomenon seems to be less pronounced when compared to both RG60 and SSG at similar values of $x / x_{\text {peak }}$. Furthermore for FSG17 the effect of vortex shedding is not even detectable in the turbulence decay region, in contrast to both RG60 and SSG at the same values of $x / x_{\text {peak }}$. We come back to this point in section 4 of this paper. The effect of vortex shedding can also be detected by directly looking at the autocorrelation coefficient $\rho_{u}$ of $u$ which is plotted in figure $8(\mathrm{~b}, \mathrm{~d}, \mathrm{f})$, where the time $t$ has been normalised by the reference time scale $t_{0} / U_{\infty}$. In the production region the coefficient $\rho_{u}$ exhibits a periodic behaviour which is damped for large $t$ and, in agreement with the previous considerations, this periodic behaviour is much less marked for FSG17. It is interesting to notice how, in the decay region, $\rho_{u}$ smoothly decays to zero with no sign of periodicity for FSG17, unlike RG60 and SSG.

The vortex shedding Strouhal numbers based on $t_{0}, S t_{s h}=f_{s h} t_{0} / U_{\infty}$, are reported in table 2 for the measurements corresponding to $U_{\infty}=5 \mathrm{~m} / \mathrm{s}$ and $U_{\infty}=17 \mathrm{~m} / \mathrm{s} ; f_{s h}$ is the frequency at which the spectra exhibit a consistent peak in the production region, at different locations along the centerline. We notice that $S t_{s h}$ is almost invariant with $U_{\infty}$ for all three grids. The value of $S t_{s h}$ for FSG17 is considerably lower when compared to both RG60 (about 24\% less) and SSG (about 33\% less). We check if, by using a different reference length for the definition of the Strouhal number, it might be possible to obtain similar vortex shedding Strouhal numbers for the different grids. For this purpose we consider the reference length $l_{0}=\sqrt{t_{0} L_{0}}$, which is proportional to 


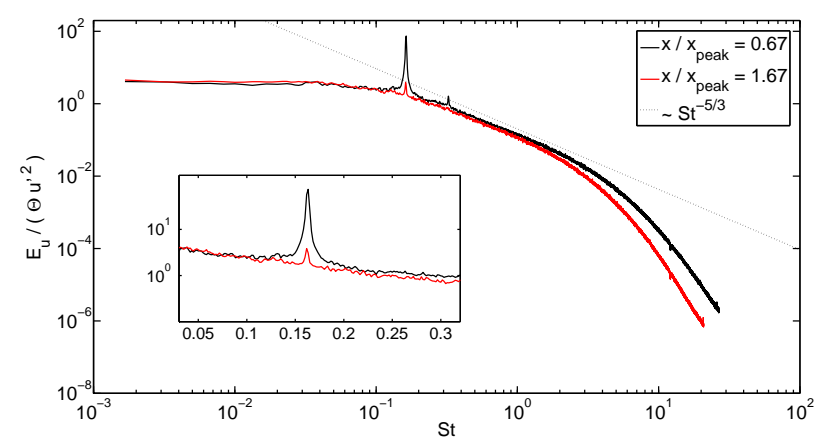

(a) RG60



(c) FSG17

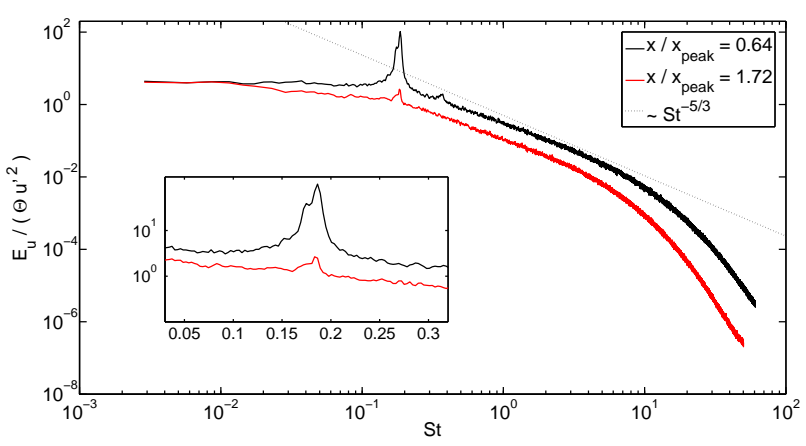

(e) SSG

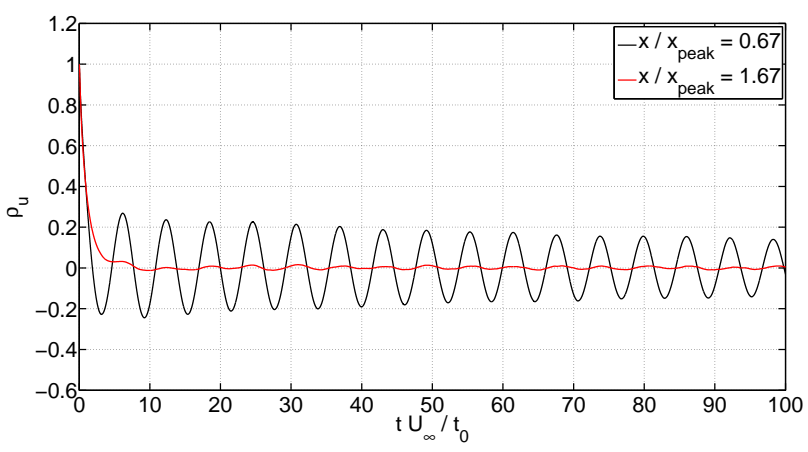

(b) RG60

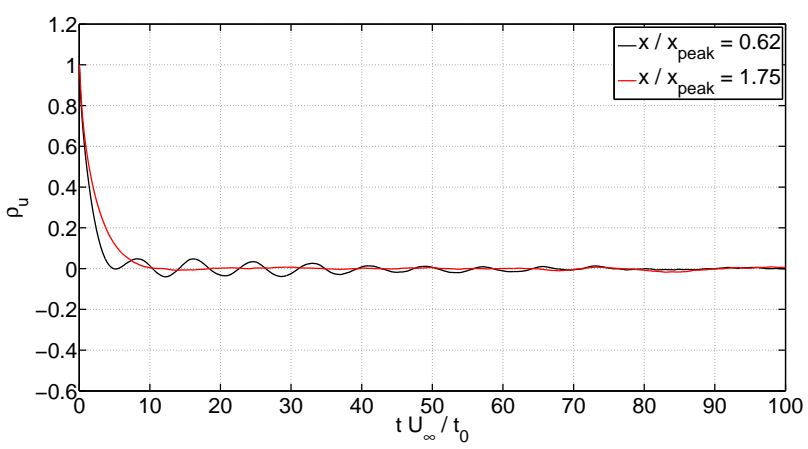

(d) FSG17

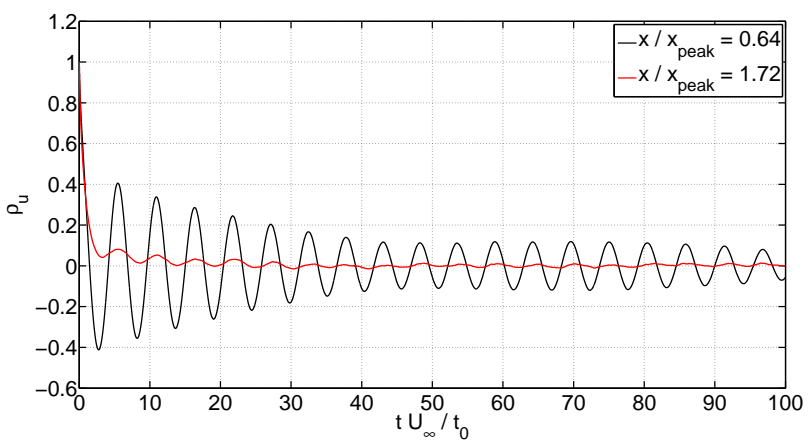

(f) SSG

Figure 8: Power spectrum density (left) and autocorrelation coefficient (right) of $u$ in the production region (black) and in the decay region (red) of RG60 (top), FSG17 (center) and SSG (bottom) on the centerline; $U_{\infty}=5 \mathrm{~m} / \mathrm{s}$.

the square root of the area of the largest bars of the grid (and also proportional to the area covered by the entire largest square pattern iteration). This approach is similar to what was done for plates with different regular and fractal geometries by Nedić et al. (2013), in accordance to the original idea of Fail et al. (1959). We define a supplementary vortex shedding Strouhal number based on $l_{0}, S t_{s h}^{A}=f_{s h} l_{0} / U_{\infty}=S t_{s h} \sqrt{L_{0} / t_{0}}$. The results in table 2 show that the values of $S t_{s h}^{A}$ for the different grids are considerably closer than for $S t_{s h}$, in particular they are almost the same for FSG17 $\left(S t_{s h}^{A} \approx 0.44\right)$ and for SSG $\left(S t_{s h}^{A} \approx 0.43\right)$. We do not have enough data to claim the universality of $S t_{s h}^{A}$ (or some closely related Strouhal number) for grid-generated turbulence. However we show that the percentage difference, between the maximum and the minimum value (with respect to the average value) among the three grids considered here, drops from $40.8 \%$ for $S t_{s h}$ to $9.5 \%$ for $S t_{s h}^{A}$.

As already mentioned in the introduction, Mazellier and Vassilicos (2010) experimentally showed that in the production region of fractal square grids the distributions of the velocity fluctuations are far from Gaussian; they exhibit high values of flatness and are highly left-skewed. In figure 9 we show the probability density functions (PDFs) of $u$ for RG60, FSG17 and SSG relative to the same previously considered streamwise positions 


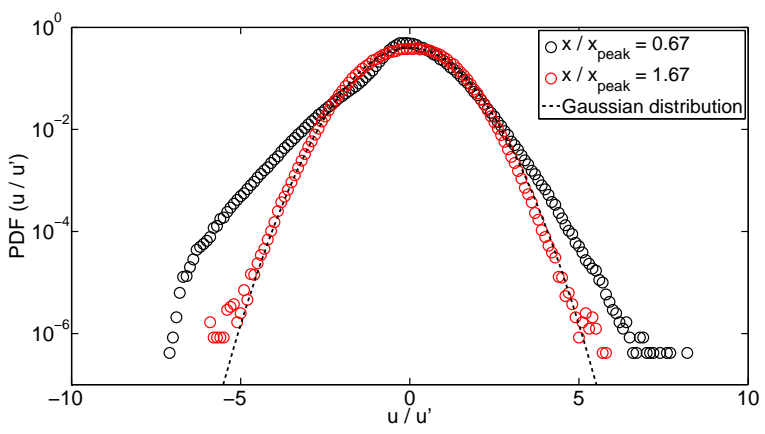

(a) RG60

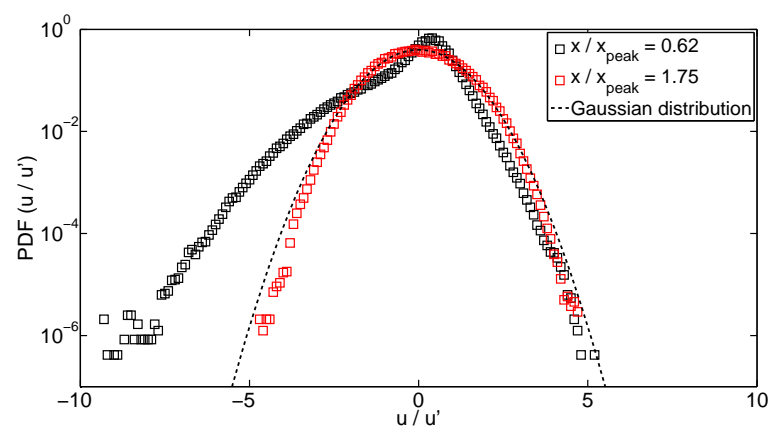

(b) FSG17

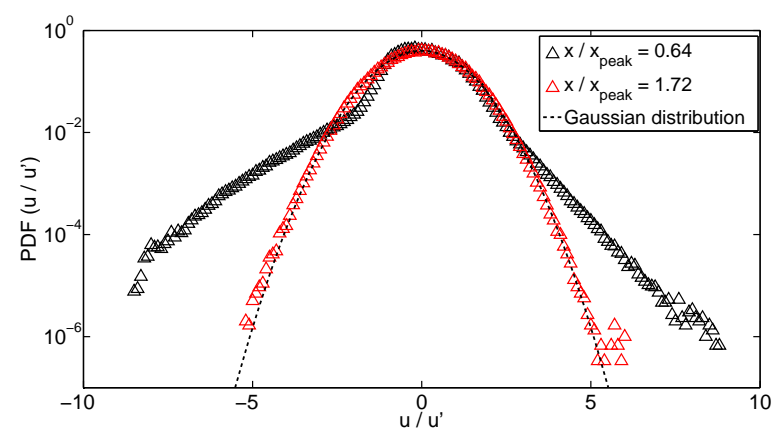

(c) SSG

Figure 9: Probability density functions of $u$ in the production region (black) and in the decay region (red) of RG60 (a), FSG17 (b) and SSG (c); $U_{\infty}=17 \mathrm{~m} / \mathrm{s}$.

(data corresponding to that plotted in figure 8). In the turbulence decay region the PDFs get close to a Gaussian distribution. For $x / x_{\text {peak }} \approx 1.7$ the skewness of $u, S=<u^{3}>/<u^{2}>^{3 / 2}$, is indeed near zero, $S=-0.09,0.07,-0.04$ and the flatness, $F=<u^{4}>/<u^{2}>^{2}$, close to $3, F=2.89,2.81,2.99$ for RG60, FSG17 and SSG respectively. On the opposite side, the PDFs clearly do not follow a Gaussian distribution and appear left-skewed in the production region $(x /$ peak $\approx 0.64)$, not only for FSG17 but also for the other two grids. The values of the skewness are indeed all negative, $S=-0.31,-1.34,-0.48$ for RG60, FSG17 and SSG respectively. The flatness exhibits values higher than $3, F=4.61,5.85,6.13$ for RG60, FSG17 and SSG respectively.

These figures suggest that, in the production region, rare strong decelerating flow events are more likely to occur than accelerating events and this holds for three turbulence-generating grids with very different geometries. However our results show that for FSG17 this feature (the high negative skewness) is even more pronounced when compared to both RG60 and SSG. Given that FSG17 is actually the grid where the vortex shedding signature appears to be less evident, it becomes natural to ask whether the energy associated with this periodic phenomenon affects the Gaussianity of the velocity fluctuations. The study of this aspect is addressed in the following section of this paper.

\section{Vortex shedding suppression}

We attempt to examine some effects of vortex shedding suppression on the flow past a turbulence-generating grid along the centerline. To pursue this goal we place a set of four splitter plates detached from SSG and we perform velocity measurements downstream of this new turbulence generator ( $\mathrm{SSG}+\mathrm{SP}$ ). We stress that our main purpose is not to optimise the vortex shedding suppression. Instead, it is to study how the flow properties change when we attenuate the vortex shedding originating from the large bars of the grid.

First we need to identify the position $x_{s p}$ of the splitter plates for which vortex shedding is more effectively suppressed. For this purpose we consider the centerline streamwise evolution of $T u$ for SSG+SP for the baseline case $U_{\infty}=11 \mathrm{~m} / \mathrm{s}$ (figure 10) with six different positions of the splitter plates, $x_{s p} / t_{0}=0,1,2,3,4,5$, and we 


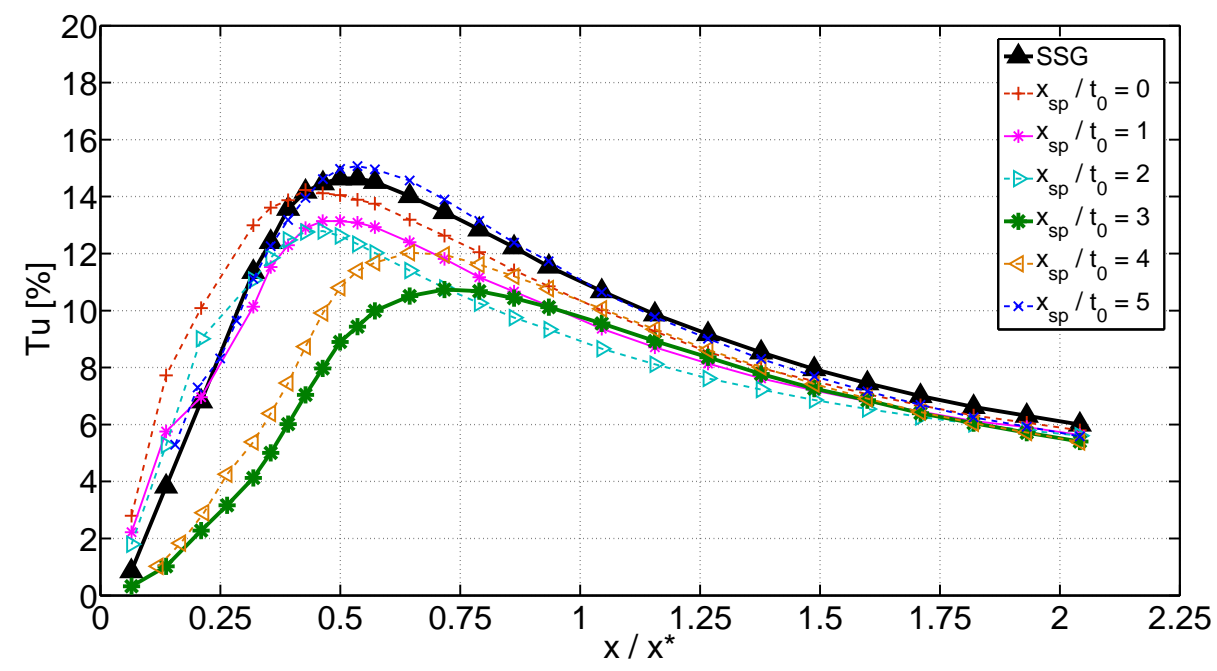

Figure 10: Turbulence intensity for SSG and SSG+SP with different values of $x_{s p} / t_{0}$ along the centerline; $U_{\infty}=11 \mathrm{~m} / \mathrm{s}$.

compare it with SSG. Among the limited number of values $x_{s p} / t_{0}$ here investigated, the position $x_{s p} / t_{0}=3$ appears to be the most effective one in decreasing the vortex shedding intensity. We motivate this statement by considering two aspects: (i) for $x_{s p} / t_{0}=3$ the distance $x_{\text {peak }}$ is maximum and (ii) the turbulence intensity at $x=x_{\text {peak }}, T u_{\text {peak }}$, is minimum. In particular, for this position of the splitter plates, $x_{\text {peak }}$ is increased by $44 \%$ when compared to the configuration without splitter plates, since it moves from $0.5 x^{*}$ to $0.72 x^{*}$. The suppression of vortex shedding causes the wakes originated from the bars of the grid to become narrower (see Anderson and Szewczyk, 1997; Akilli et al., 2005; Chen and Shao, 2013) and therefore we postulate that the location of the peak of turbulence intensity, which is representative of the location where the wakes meet (Mazellier and Vassilicos, 2010), is moved downstream. This also means that the drag coefficient $c_{d}$ of the bars is reduced. If we consider the scaling $x_{\text {peak }} / x^{*} \propto c_{d}^{-1}$, an increase of $44 \%$ in $x_{p e a k} / x^{*}$ can be explained by a decrease in $c_{d}$ of about $31 \%$, which we cannot directly assess since we do not measure the drag of the bars. However, in order to check the consistency of our findings, we can additionally make use of the scaling for $T u_{\text {peak }}, T u_{\text {peak }} \propto c_{d} t_{0} / L_{0}$. By considering this last relation, we would theoretically expect that $T u_{\text {peak }}$ is also reduced by about $31 \%$ due to the reduction in $c_{d}$. Looking at our baseline case for SSG at $U_{\infty}=11 \mathrm{~m} / \mathrm{s}$ for consistency, we find that for $x_{s p} / t_{0}=3$ the value of $T u_{\text {peak }}$ decreases from 0.146 to 0.107 , a reduction of $27 \%$. Moreover, $x_{s p} / t_{0}=3$ is close to $x_{s p} / D=2.5-2.7$, which was found to be the optimal distance for suppressing vortex shedding from a circular cylinder (see Roshko, 1954; Lin and Wu, 1994; Hwang et al., 2003).

Given that $x_{s p} / t_{0}=3$ proves to be the most effective distance for suppressing vortex shedding (from the limited number of positions here tested), we now focus on this position only for the remainder of this paper and we refer to this configuration as SSG+SP3. In figure 11 we show the normalised mean velocity and the turbulence intensity along the centerline for SSG+SP3. One can see that both the mean velocity and the turbulence intensity profiles at $U_{\infty}=11 \mathrm{~m} / \mathrm{s}$ and $17 \mathrm{~m} / \mathrm{s}$ are very well collapsed. The ratio $U / U_{\infty}$ for $U_{\infty}=5 \mathrm{~m} / \mathrm{s}$ is found to be slightly lower with respect to the measurements at higher inlet velocities, with a maximum difference of $3.8 \%$ at $x / x^{*}=0.64$. However the discrepancy becomes attenuated and tends to disappear as one proceeds downstream, similarly to what was found for SSG (see figure 4a). Comparison of Figure 4a (SSG) and figure 11a (SSG+SP3) shows that the mean velocity for $0.25<x / x^{*}<0.6(0.3 \mathrm{~m}$ $<x<0.7 \mathrm{~m}$ ) is higher for SSG+SP3. This can be explained by the presence of the four splitter plates which create a contraction effect and therefore an acceleration of the flow. The turbulence intensity for $U_{\infty}=5 \mathrm{~m} / \mathrm{s}$ is marginally higher just in the proximity of $x_{\text {peak }}$, whose location remains the same for all the measurements performed, $x_{\text {peak }}=0.72 x^{*}$. 


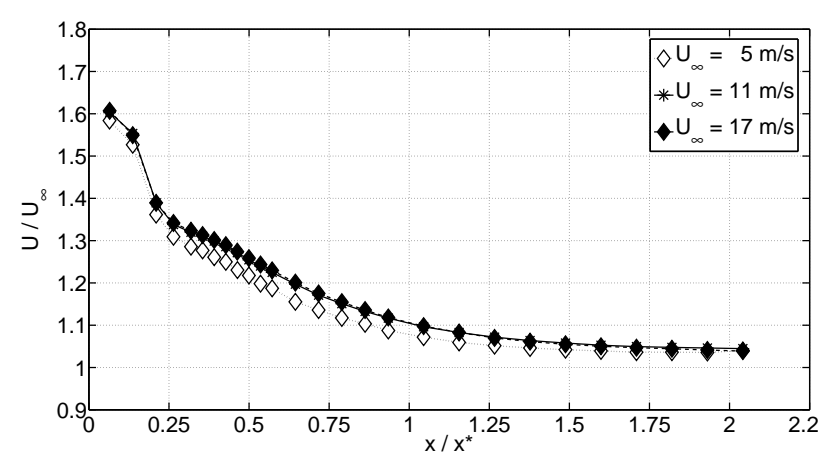

(a)

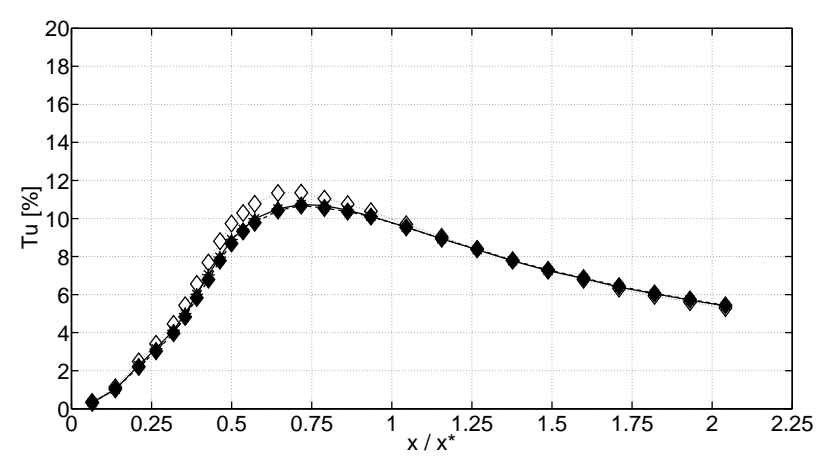

(b)

Figure 11: Mean velocity (a) and turbulence intensity (b) for SSG+SP3 along the centerline.

\subsection{Vortex shedding energy}

We are interested in comparing the energy related to the vortex shedding downstream of the different grids. In this work we refer to vortex shedding energy, $E_{s h}$, as the portion of the turbulent kinetic energy $u^{\prime 2}$ which is associated with a frequency bandwidth centred around $f_{s h}$. In the production region, the main contribution to $E_{s h}$ is due to vortex shedding. However it must be noted that $E_{s h}$ contains also part of the energy associated with the turbulent stochastic motion. The stochastic contribution can also be significant, especially past the wakes's interaction's location, given that vortex shedding occurs as a low-frequency (large scale) phenomenon.

In figure 12 we show the contour plots of the power spectrum density $E_{u}$ for RG60, FSG17, SSG and SSG+SP3 along the centerline for $0.35 \leq x / x_{\text {peak }} \leq 2$ and $0.04<S t<1.1$. The values of $E_{u}$ are normalised using the local $u^{\prime 2}$ as a reference energy and $U_{\infty} / T$ as a reference frequency (we do this not to contaminate the values with $t_{0}$ or $L_{0}$ which can be different for our grids). When considering the case of SSG in the production region $\left(x / x_{\text {peak }}<1\right)$, one can see that a significant contribution to the total kinetic energy comes from a narrow range of Strouhal numbers across $S t_{s h}$, i.e. it is mainly due to vortex shedding. The signature of vortex shedding is stronger and more persistent for SSG when compared to all the other turbulence-generating grids at the same $x / x_{\text {peak }}$. On the opposite side the vortex shedding energy contribution to $u^{\prime 2}$ appears to be the lowest for FSG17. Moreover, for FSG17 the effect of vortex shedding disappears more quickly in terms of $x / x_{p e a k}$ as, unlike all other configurations, it is not even detectable for about $x / x_{\text {peak }}>0.8$. Given these qualitative figures, we can already argue that the higher values of $T u$ for SSG with respect to FSG17 (in the production region and close to $x_{\text {peak }}$ ) can be explained physically by a more significant vortex shedding contribution.

In figure 13 we compare the spectra of $u$ in the production region for SSG and SSG+SP3, specifically at $x / x_{\text {peak }}=0.7$. By making use of $\Theta u^{\prime 2}$ to normalise $E_{u}$, the spectra for the two configurations are very well collapsed with the exception of the frequency range which lies in the proximity of the vortex shedding frequency. From figures 12 and 13 we can observe three main effects due to the addition of the splitter plates downstream of SSG (SSG+SP3): (i) the vortex shedding contribution to $u^{\prime 2}$ decreases, (ii) the vortex shedding signature on the centerline is less persistent, (iii) vortex shedding appears as a more broad-band phenomenon and therefore the energy is re-distributed among a broader range of frequencies (scales). Given this last aspect, for SSG+SP3 it is not possible to identify a frequency $f_{s h}$ at which a clear peak in the spectra can be observed. For this reason, in order to define a vortex shedding Strouhal number $S t_{s h}$ for SSG+SP3, we consider the frequency $f_{s h}$ where a local energy maximum is present. With respect to SSG (see table 2), for SSG+SP3 the value of $S t_{s h}$ slightly decreases. We find $S t_{s h}=0.182$ for $U_{\infty}=11 \mathrm{~m} / \mathrm{s}$ and $17 \mathrm{~m} / \mathrm{s}$ and $S t_{s h}=0.172$ for $U_{\infty}=5 \mathrm{~m} / \mathrm{s}$.

In order to give an estimate of the reduction of the vortex shedding energy due to the presence of the splitter plates, we follow Nedić et al. (2015). We compute the vortex shedding energy $E_{s h}$ by integrating the power spectrum density $E_{u}$ for an interval of Strouhal numbers $\Delta S t$ centred across $S t_{s h}$ :

$$
E_{s h}(\Delta S t)=\int_{S t_{1}}^{S t_{2}} E_{u}(S t) d S t
$$




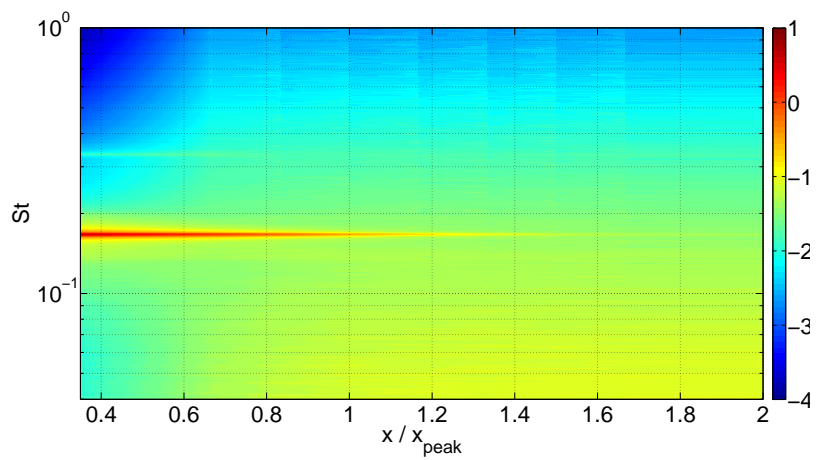

(a) RG60

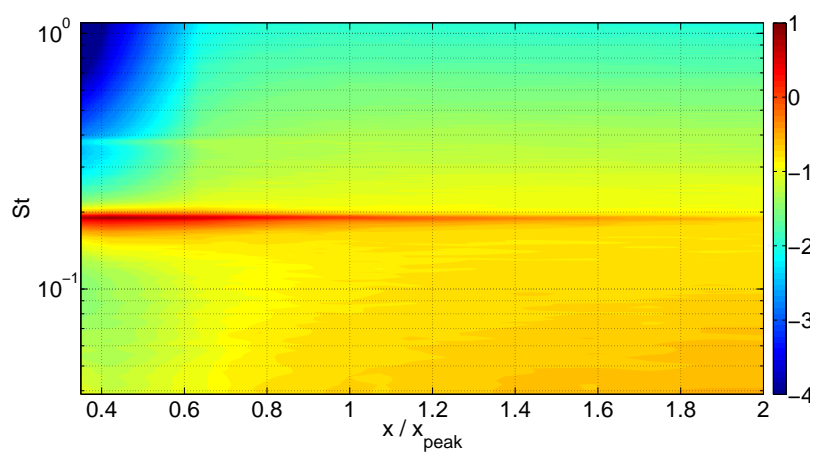

(c) SSG

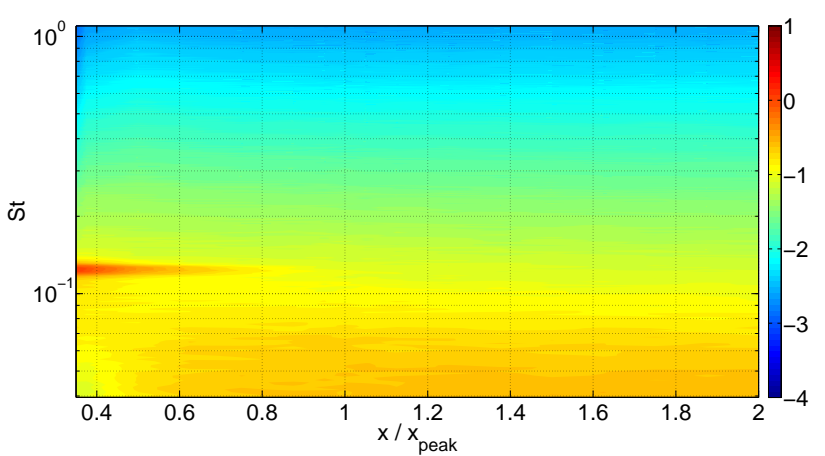

(b) FSG17

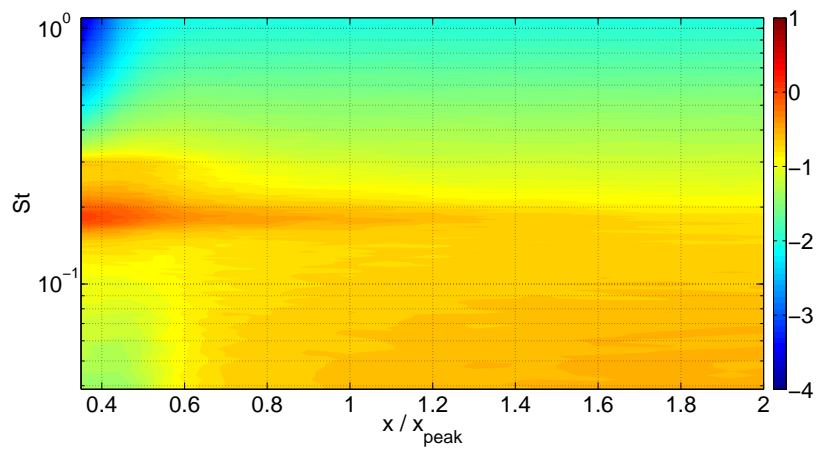

(d) $\mathrm{SSG}+\mathrm{SP} 3$

Figure 12: Contour plots (in logarithmic scale) of the power spectrum density, normalised by $u^{\prime 2} T / U_{\infty}$, along the centerline for RG60 (a), FSG17 (b), SSG (c) and SSG+SP3 (d); $U_{\infty}=17 \mathrm{~m} / \mathrm{s}$.

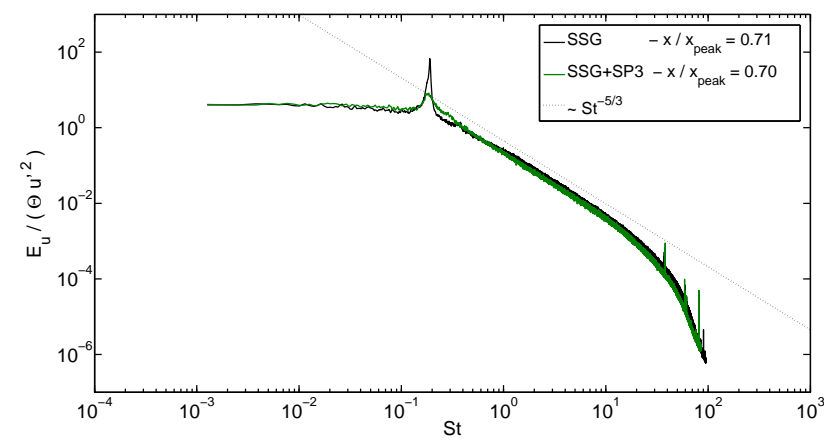

(a)

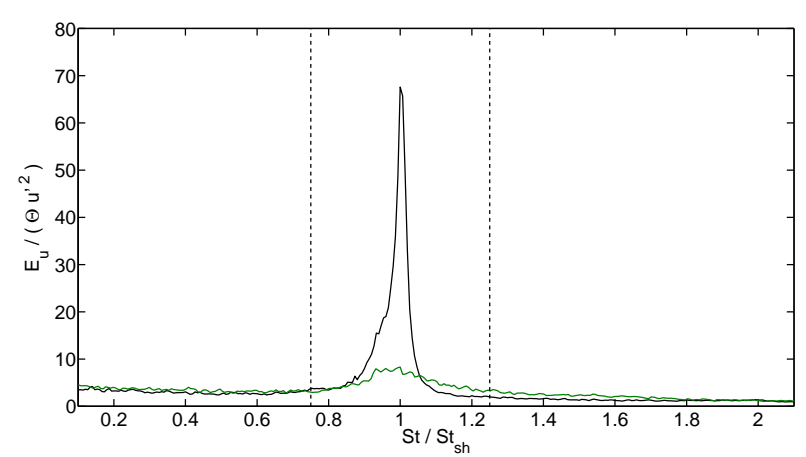

(b)

Figure 13: Power spectrum density of $u$ in logarithmic (left) and linear (right) scale for SSG (black) and SSG+SP3 (green) at $x / x_{\text {peak }}=0.7 ; U_{\infty}=17 \mathrm{~m} / \mathrm{s}$. The vertical dashed lines in (b) identify the interval $\Delta S t / S t_{s h}=0.5$ centred at $S t_{s h}$.

where $S t_{1}=S t_{s h}-\Delta S t / 2$ and $S t_{2}=S t_{s h}+\Delta S t / 2$. It is important to notice that the value of $E_{s h}$ depends on the arbitrary choice of $\Delta S t$, therefore it is required to check how $E_{s h}$ varies for different $\Delta S t$.

We quantify $E_{s h}$ for increasing values of $\Delta S t / S t_{s h}$ and for $x / x^{*} \leq 0.72$ (extent of the production region for $\mathrm{SSG}+\mathrm{SP} 3$ ); we use $E_{s h}^{I}$ (figure 14a) to refer to the original configuration (SSG) and $E_{s h}^{I I}$ (figure 14b) for the configuration with the four splitter plates $(\mathrm{SSG}+\mathrm{SP} 3)$. The quantity $E_{s h}$ obviously increases with $\Delta S t$ according to equation 5 . However, for the same streamwise location and for the same $\Delta S t / S t_{s h}, E_{s h}$ is always lower for the configuration with the splitter plates. The ratio $E_{s h}^{I I} / E_{s h}^{I}$ (figure 14c) is indeed always less than 1 in the entire turbulence production region of SSG+SP3, thus confirming the vortex shedding attenuation. The reduction is greater for lower values of $x$, that is where vortex shedding is more prominent. It is interesting to notice that for $\Delta S t / S t_{s h} \gtrsim 0.5$ the ratio $E_{s h}^{I I} / E_{s h}^{I}$ remains approximately constant. This result allows us to 


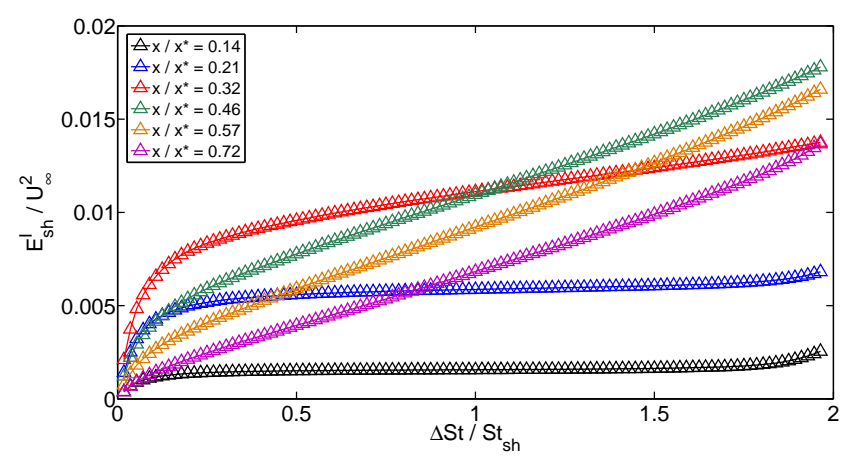

(a)

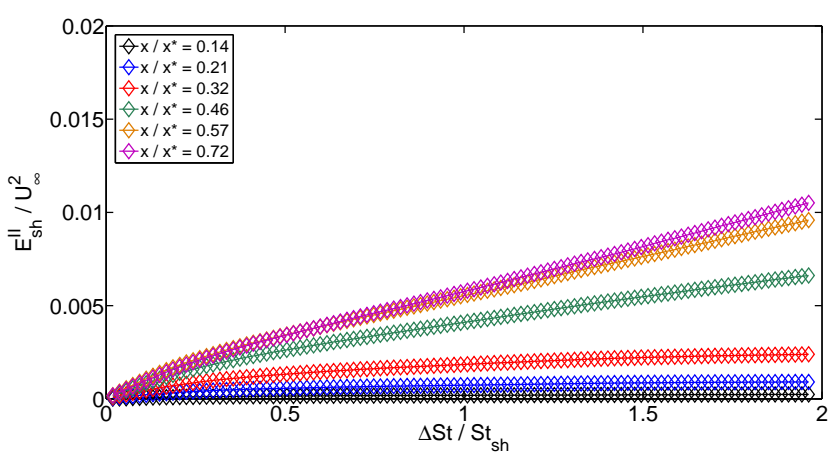

(b)

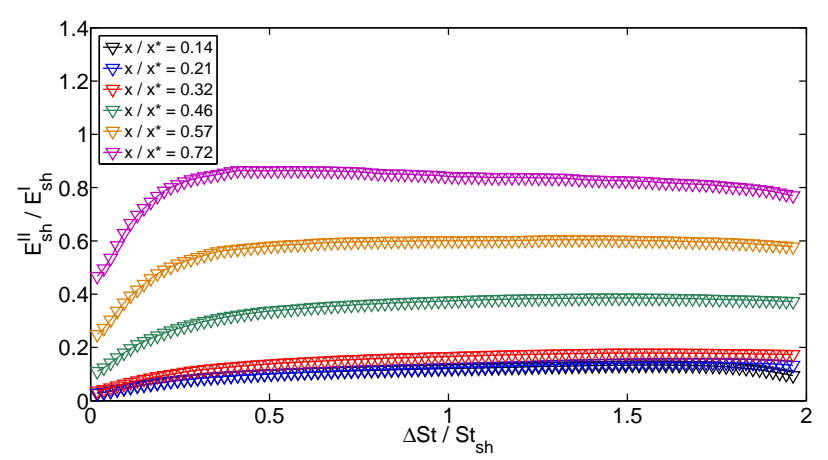

(c)

Figure 14: Vortex shedding energy $E_{s h}$ for SSG (a), for SSG+SP3 (b) and ratio between the two (c) varying $\Delta S t / S t_{s h}$ at different streamwise locations along the centerline; $U_{\infty}=17 \mathrm{~m} / \mathrm{s}$.

quantify the vortex shedding suppression along the centerline without having to deal with a strong dependence on $\Delta S t$. For this reason we choose the particular value $\Delta S t / S t_{s h}=0.5$ for the integration of the power spectrum density (see figure 13b) for the comparisons in figure 15 , where $E_{s h}$ is taken to be $E_{s h}\left(\Delta S t / S t_{s h}=0.5\right)$.

Figure 15a shows the evolution of $E_{s h}$ along $x / x^{*}$ for SSG and SSG+SP3. Similarly to the profiles of $T u$, $E_{s h}$ first increases with $x$, reaches a peak value and then subsequently decreases. For $x / x^{*}<0.32$ this energy increases with a very steep gradient in the case of SSG, whereas in the same region the increase is attenuated for SSG+SP3. For $x / x^{*}>0.85$ the profiles of $E_{s h}$ for SSG and SSG+SP3 collapse. However we have to point out that in this region the vortex shedding signature has almost disappeared, therefore in this case $E_{s h}$ loses the meaning of vortex shedding energy. The streamwise position where $E_{s h}$ is maximum anticipates the peak of turbulence intensity (figure 15b) more evidently for SSG. The maximum value of $E_{\text {sh }}$ occurs at $x / x_{\text {peak }}=0.9$ for SSG+SP3 and at $x / x_{\text {peak }}=0.7$ for SSG.

The percentage reduction of $E_{s h}$ for $\mathrm{SSG}+\mathrm{SP} 3\left(E_{s h}^{I I}\right)$ with respect to $\mathrm{SSG}\left(E_{s h}^{I}\right)$, reaches almost $80 \%$ at $x / x_{\text {peak }}=0.45$ (figure 15c). The reduction decreases further downstream until $x / x_{\text {peak }}=1$ where it is about $50 \%$ and it remains approximately around this value for larger distances from the grid. The diminution of $E_{s h}$ occurs also when compared to the total turbulent kinetic energy (figure $15 \mathrm{~d}$ ). For $\Delta S t / S t_{s h}=0.5$, the maximum value of $E_{s h} / u^{\prime 2}$ is about $80 \%$ for SSG at $x / x_{\text {peak }}=0.42$, whereas for SSG+SP3 the maximum occurs at $x / x_{\text {peak }}=0.29$ where $E_{s h} / u^{\prime 2}$ is about $60 \%$. Following these positions there exists a region until $x / x_{\text {peak }}=1$, where the ratio $E_{s h} / u^{\prime 2}$ is substantially lower for SSG+SP3. For example at $x / x_{\text {peak }}=0.64$ the value of this ratio is $54 \%$ for SSG and $27 \%$ for SSG+SP3. In the same figure (figure $15 \mathrm{~d}$ ) we also plot the ratio $E_{s h} / u^{\prime 2}$ for FSG17 with the same choice of $\Delta S t / S t_{s h}$, i.e. $\Delta S t / S t_{s h}=0.5$. For $x / x_{\text {peak }}>0.35, E_{s h} / u^{\prime 2}$ is considerably lower for FSG17 when compared both to SSG and to SSG+SP3. In the turbulence production region for the grid FSG17, which has $\sigma=25 \%$, the vortex shedding energy is lower than for SSG which has almost the same $L_{0}$ and $\sigma=20 \%$, with this energy being also lower than for SSG+SP3. The interaction between wakes of different size, which occurs only with FSG17, could be an explanation for the weaker vortex shedding. 


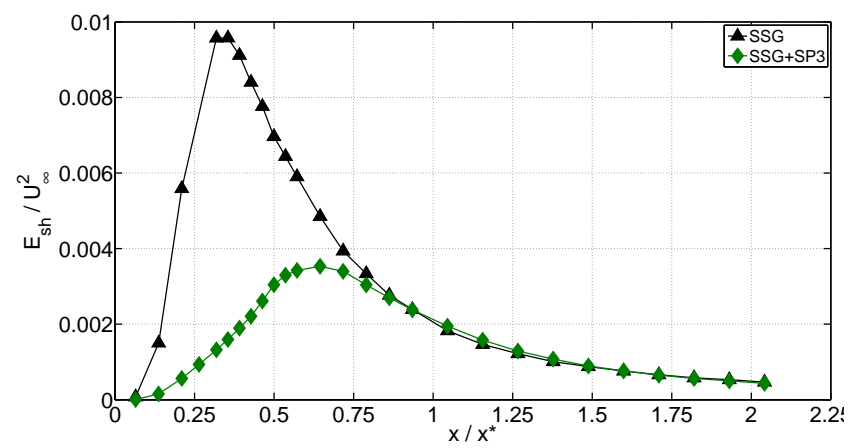

(a)

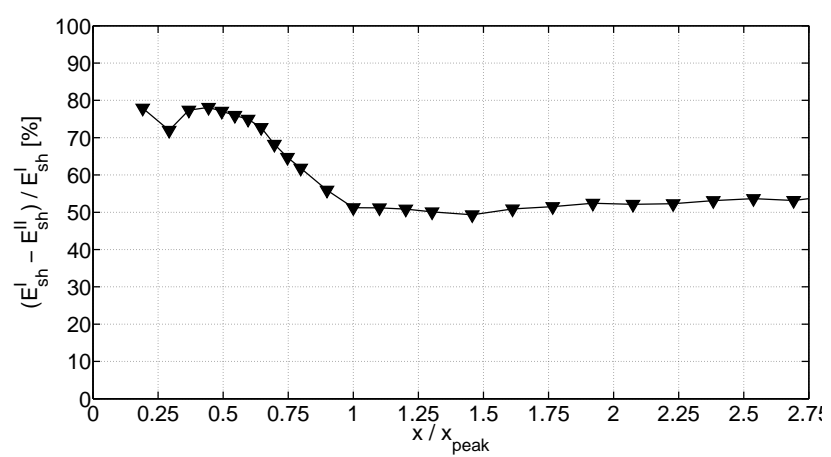

(c)

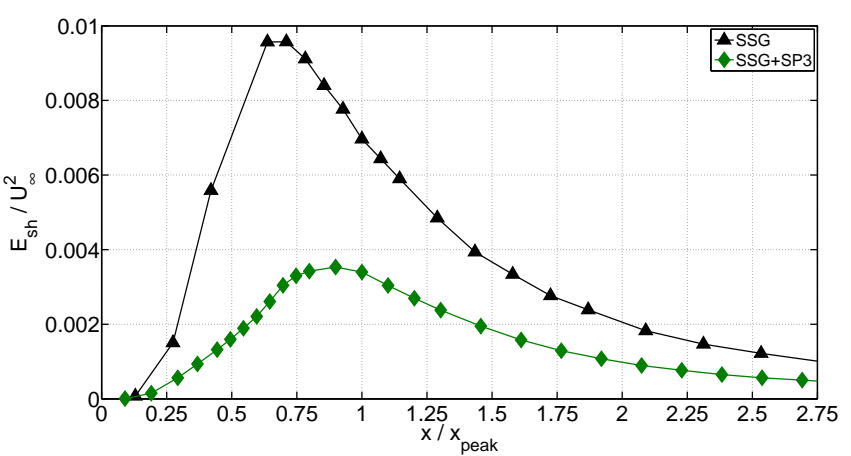

(b)

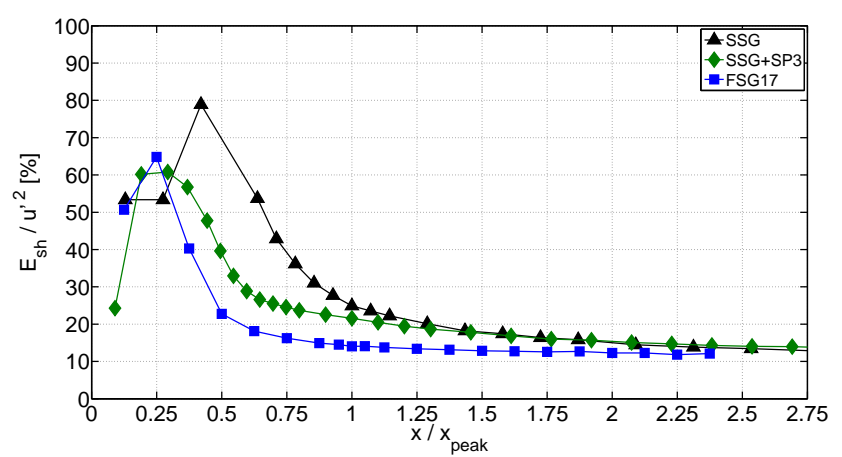

(d)

Figure 15: Vortex shedding energy $E_{\text {sh }}$ for SSG and SSG+SP3 as a function of $x / x^{*}$ (a) and of $x / x_{p e a k}$ (b), percentage reduction of $E_{s h}$ for SSG+SP3 with respect to SSG (c), share of turbulent kinetic energy due to vortex shedding for SSG, SSG+SP3 and FSG17 (d); $\Delta S t / S t_{s h}=0.5, U_{\infty}=17 \mathrm{~m} / \mathrm{s}$.

\subsection{Effects of vortex shedding}

The effects induced by the presence of the splitter plates on the downstream evolution of the turbulence length scales are examined along the centerline. The integral length scale $L_{u}$ (figure 16a) takes very similar values for $\mathrm{SSG}$ and SSG+SP3 in the range $0.5<x / x^{*}<1$. However, when considering the turbulence decay regions, it is clearly noticeable that the growth of $L_{u}$ with $x$ is reduced for SSG+SP3. We remark that the range where this occurs $\left(x / x^{*}>0.5\right)$ is quite far from the splitter plates, whose trailing edge is at $x / x^{*}=0.16$ for the SSG+SP3 configuration. This result is analogous to what we have observed for FSG17 (where vortex shedding is also reduced), for which the increase of $L_{u}$ with $x$ is also found to be slower than that for SSG (see figure 7a).

Similarly to $L_{u}$, the growth of the Taylor microscale $\lambda$ along the streamwise direction (figure 16b) is also lower for the configuration with the splitter plates. In particular, in the decay region, $\partial \lambda / \partial x \approx \partial L_{u} / \partial x$ for both SSG and SSG+SP3. The last condition is actually required in order to satisfy $L_{u} / \lambda \approx$ constant along $x$ in the $C_{\varepsilon} \neq$ constant region of turbulence (see Vassilicos, 2015). We show in fact that, even when we add the splitter plates, the ratio $L_{u} / \lambda$ remains approximately constant (figure 16c) in the region where $R e_{\lambda}$ is decreasing (figure 16d). Analogously the coefficient $C_{\epsilon}$ (figure 16e) is not constant, but it instead increases with downstream distance in the turbulence decay region of SSG and of SSG+SP3, where $R e_{\lambda}$ is decreasing. Mazellier and Vassilicos (2010) found that, in the turbulence decay region of fractal square grids, the value of $L_{u} / \lambda$ grows with the inlet Reynolds number $R e_{t_{0}}$. In our experiment we find that, when considering SSG and SSG+SP3 separately, $L_{u} / \lambda$ increases with $U_{\infty}$ and therefore with $R e_{t_{0}}$. This is a consequence of the fact that $\lambda$ reduces for increasing $U_{\infty}\left(L_{u}\right.$ is not significantly dependent on $\left.U_{\infty}\right)$. Nevertheless it is interesting to notice that the values of $L_{u} / \lambda$ are different between SSG and SSG+SP3 even though $U_{\infty}$ and $t_{0}$ (and therefore $R e_{t_{0}}$ ) are the same. The presence of the splitter plates at the very beginning of the production region modifies the streamwise development of the wakes originating from the bars of SSG (as we have seen for example from the increase in $x_{\text {peak }}$ ) and it affects the evolution of the turbulence scales along the centerline. In our case SSG and 


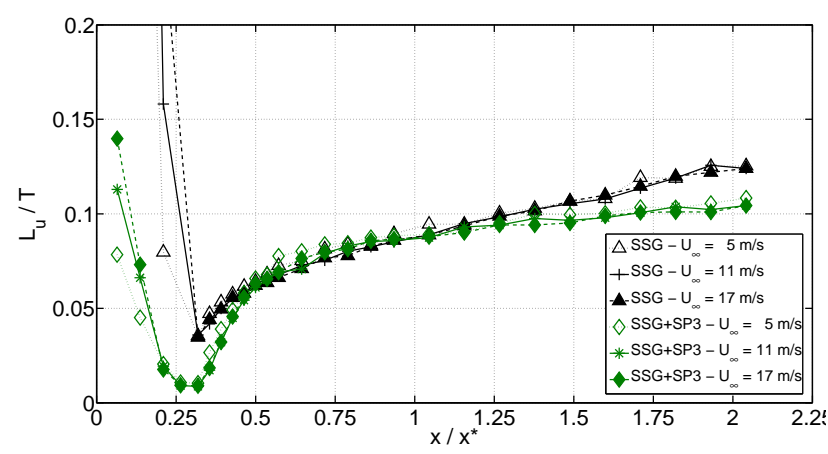

(a)

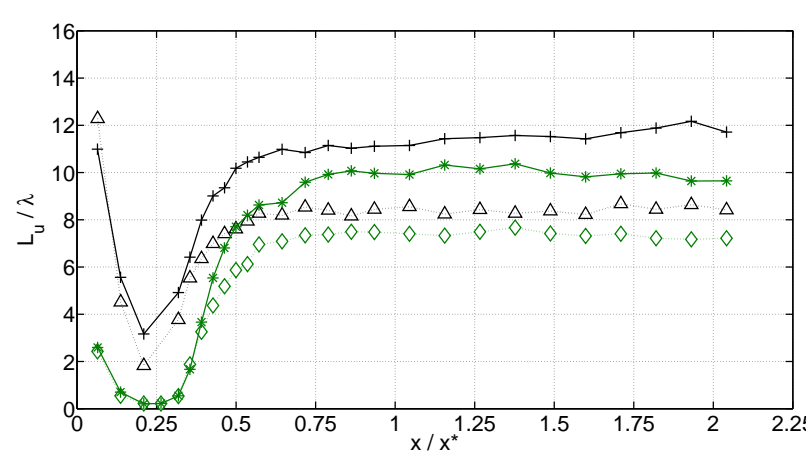

(c)

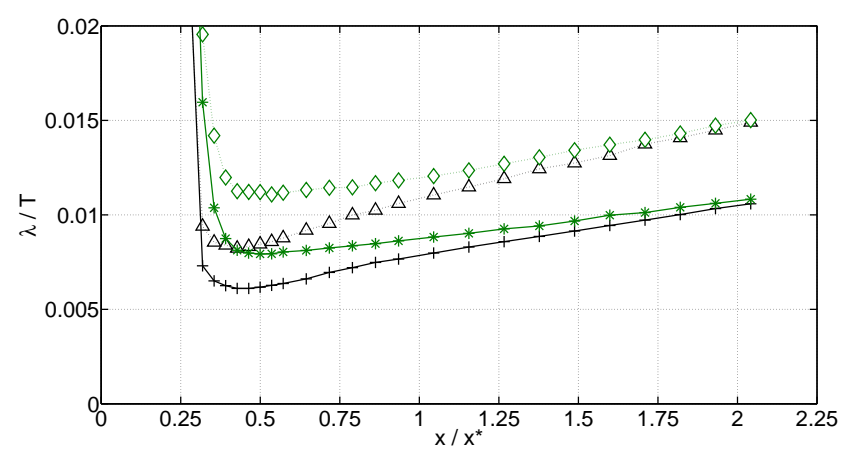

(b)

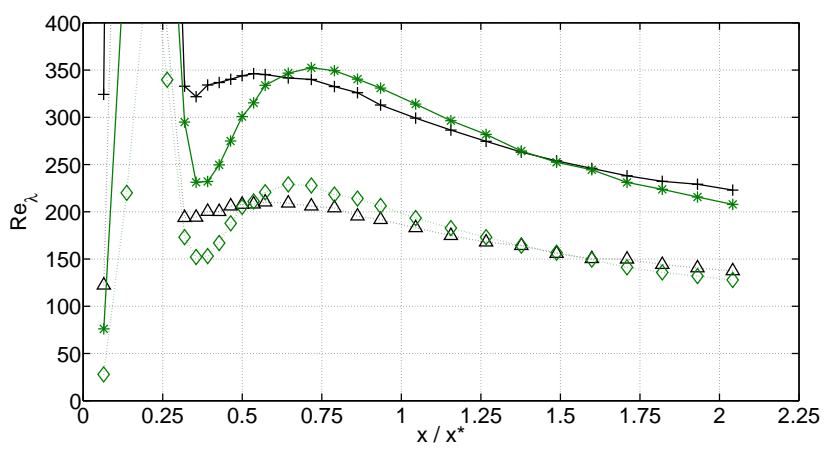

(d)

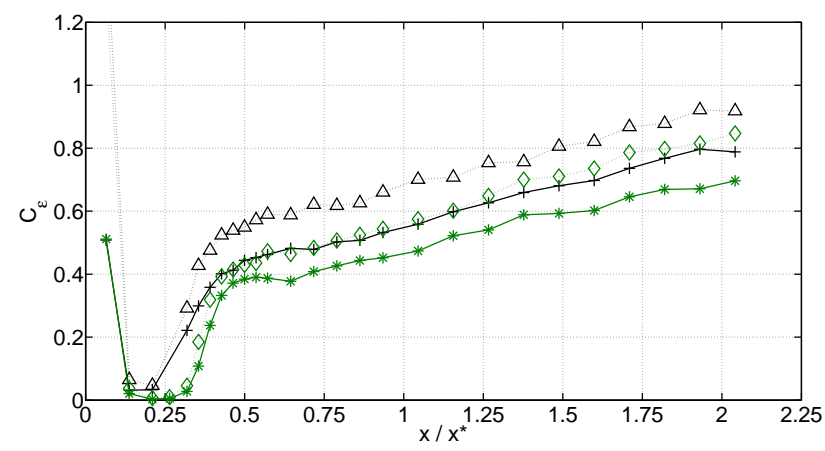

(e)

Figure 16: Integral length scale (a), Taylor length scale (b), ratio between integral and Taylor length scales (c), Reynolds number based on Taylor length scale (d) and dissipation coefficient (e) for SSG (black) and SSG+SP3 (green) along the centerline. Data for $U_{\infty}=17 \mathrm{~m} / \mathrm{s}$ are not shown for quantities derived from $\varepsilon$ as explained in section 2.4.

$\mathrm{SSG}+\mathrm{SP} 3$ can be considered as two different turbulence-generators and in particular we find that, by adding the splitter plates, the ratio $L_{u} / \lambda$ decreases.

We are interested in studying if and how the higher order statistics of $u$ are influenced by the vortex shedding suppression. We first plot the skewness $S$ (figure 17a) and the flatness $F$ (figure 17c) of $u$ for SSG+SP3 at different values of $U_{\infty}$. There is a good collapse between the results for the three inlet velocities. This ensures that $S$ and $F$ have no Reynolds dependence, at least in the range here investigated. The skewness is initially negative in the turbulence production region, reaching a minimum value around $x / x^{*}=0.45$, before increasing further downstream and becoming positive in the far decay region, where it assumes small yet non-zero values, this being a typical feature of decaying grid-generated turbulence (Maxey, 1987). The flatness steeply increases in the production region, where it exhibits a peak around $x / x^{*}=0.4$, and subsequently decreases towards values close to 3 in the decay region $\left(x / x^{*}>0.8\right)$.

At this point we compare the evolutions of $S$ and $F$ along the centerline for both SSG and SSG+SP3. The 


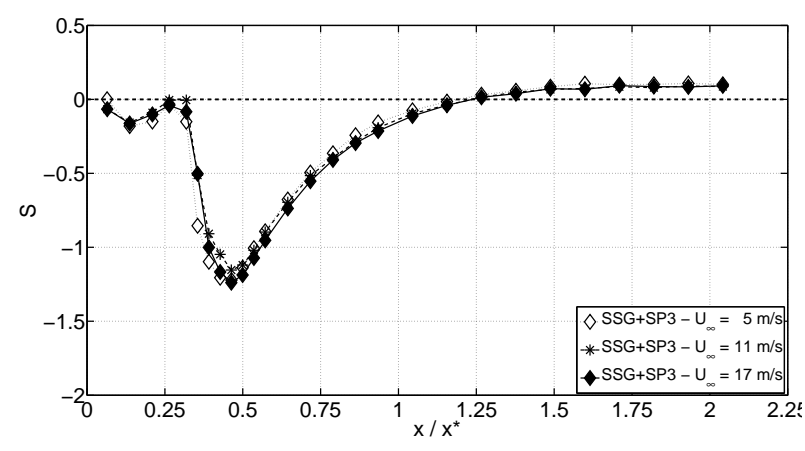

(a)

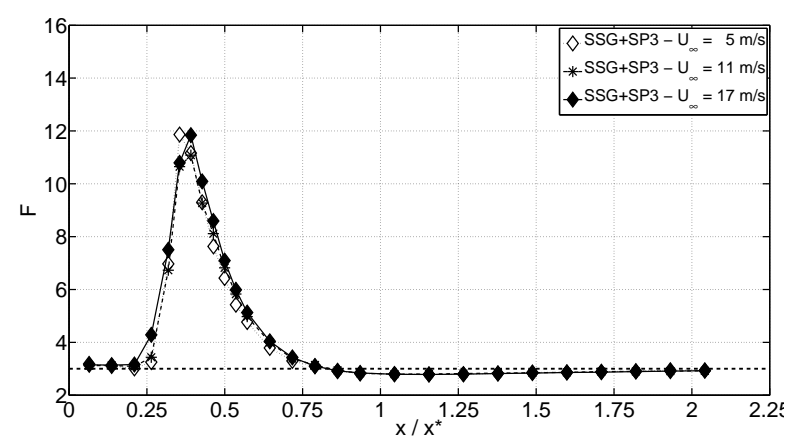

(c)

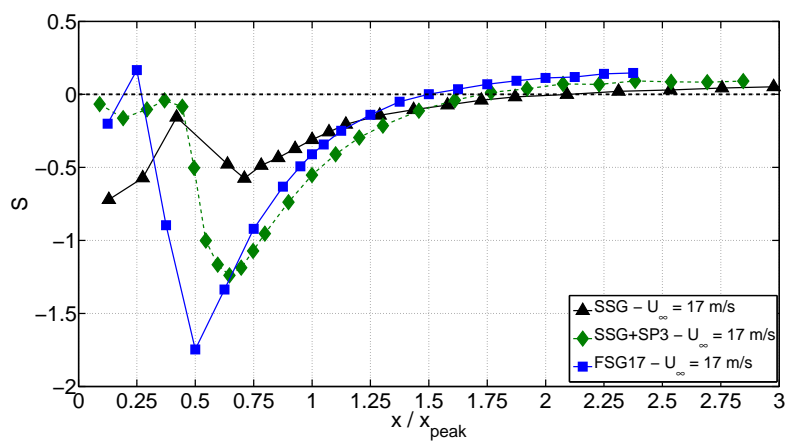

(b)

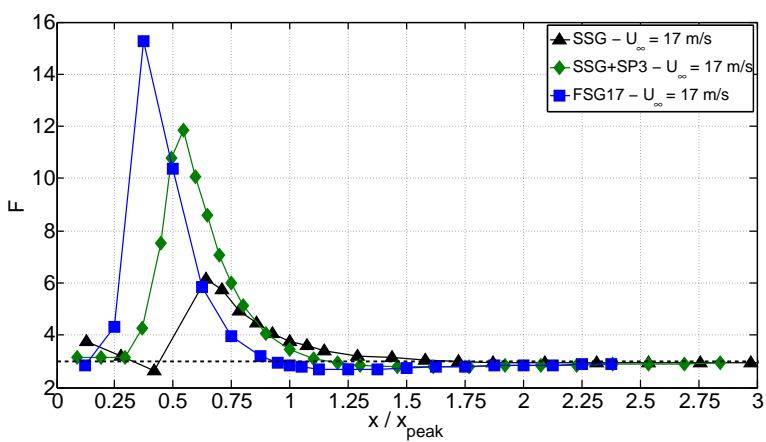

(d)

Figure 17: Skewness (top) and Flatness (bottom) for SSG+SP3 at $U_{\infty}=5,11,17 \mathrm{~m} / \mathrm{s}$ as a function of $x / x^{*}$ (left) and comparison with SSG and FSG17 for $U_{\infty}=17 \mathrm{~m} / \mathrm{s}$ as a function of $x / x_{\text {peak }}$ (right). The horizontal dashed lines identify $S=0$ (top) and $F=3$ (bottom).

streamwise positions where the skewness (figure 17b) is minimum and the flatness (figure $17 \mathrm{~d}$ ) is maximum are very similar between SSG and SSG+SP3 in terms of $x / x_{\text {peak }}$. The most striking differences between the two configurations is that for SSG+SP3 (i) the skewness reaches more negative values in the production region and (ii) the flatness is higher. We also consider the results for FSG17, for which $E_{s h}$ is the lowest, and we plot $S$ and $F$ for this grid in the same figures. We find that the absolute values of the skewness and the flatness reach the highest values for FSG17. Our results would suggest that an effect of vortex shedding in grid-generated turbulence is to "hide" the non-Gaussian behaviour of $u$ in the production region. In fact, when vortex shedding is highly energetic, $S$ and $F$ get closer to values typical of a Gaussian distribution, i.e. $S \rightarrow 0$ and $F \rightarrow 3$, in the production region too. In appendix B we perform a simple exercise to show that an increase in the vortex shedding energy is consistent with a decrease in the absolute value of the skewness of $u$.

\subsubsection{Wavelet transform in production and decay regions}

In order to support our last observations on the Gaussianity of $u$, we look directly at the effects of vortex shedding on the statistics associated with the frequencies in the proximity of $f_{s h}$. For this purpose we perform a continuous wavelet transform of velocity signals obtained for all our four turbulence generators. This type of transform allows to analyse the relative contributions of the scales a (time-scales of dilatation) to the signal $u(t)$ at instants $t^{\prime}$. The wavelet transform $u_{\Psi}\left(a, t^{\prime}\right)$ of $u(t)$ is defined as (Farge, 1992):

$$
u_{\Psi}\left(a, t^{\prime}\right)=a^{-1 / 2} \int u(t) \Psi^{*}\left(\frac{t-t^{\prime}}{a}\right) d t
$$

where $\Psi$ is the "mother" wavelet function ( $\Psi^{*}$ is its complex conjugate). The time scales $a$ can be converted to (pseudo-) frequencies $f$ by taking into account the center frequency $f_{c}$ at which the magnitude of the Fourier transform of $\Psi$ is maximum. In our context we basically use this type of transform as a systematic way to apply 


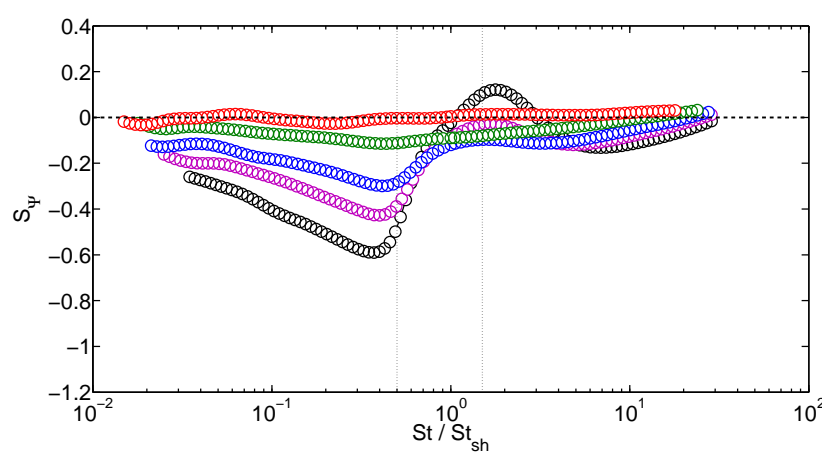

(a) RG60

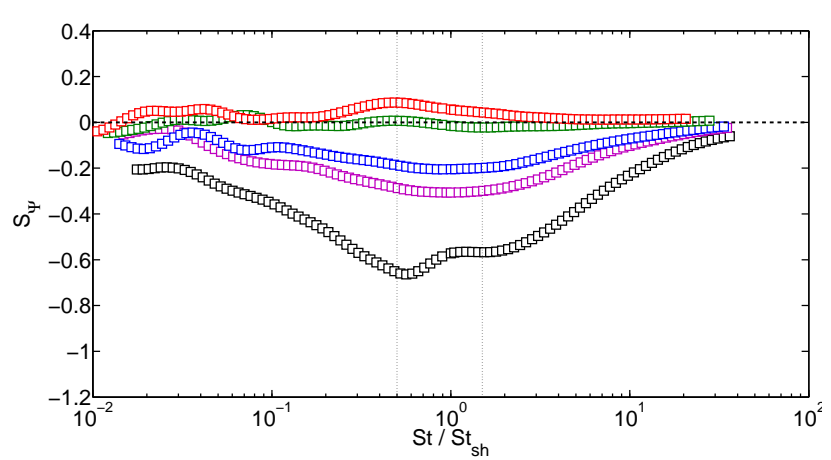

(c) FSG17

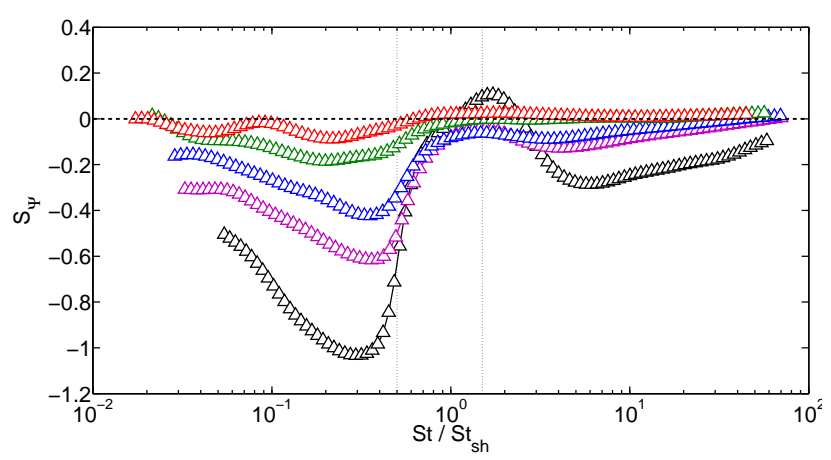

(e) SSG

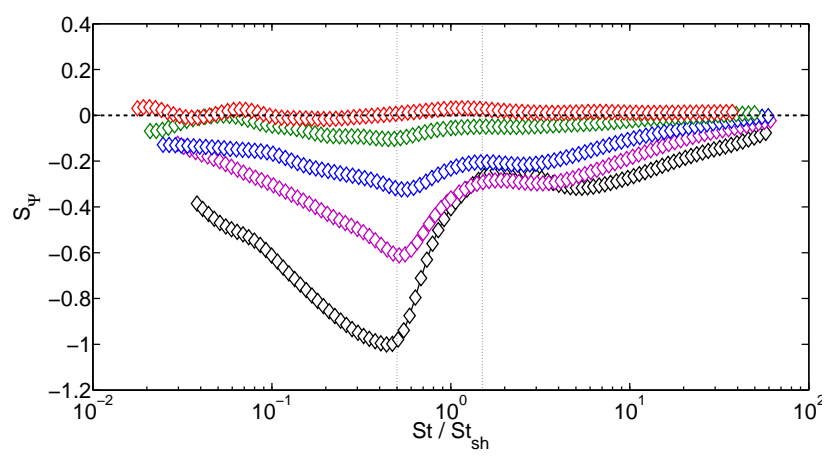

(g) $\mathrm{SSG}+\mathrm{SP} 3$

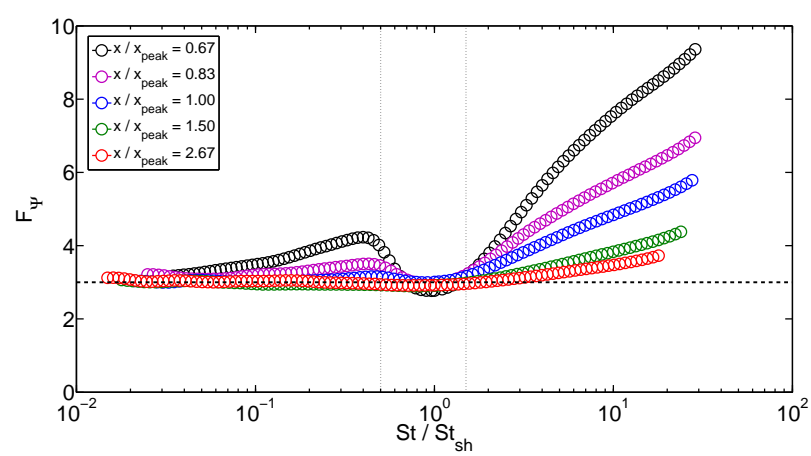

(b) RG60



(d) FSG17



(f) $\mathrm{SSG}$

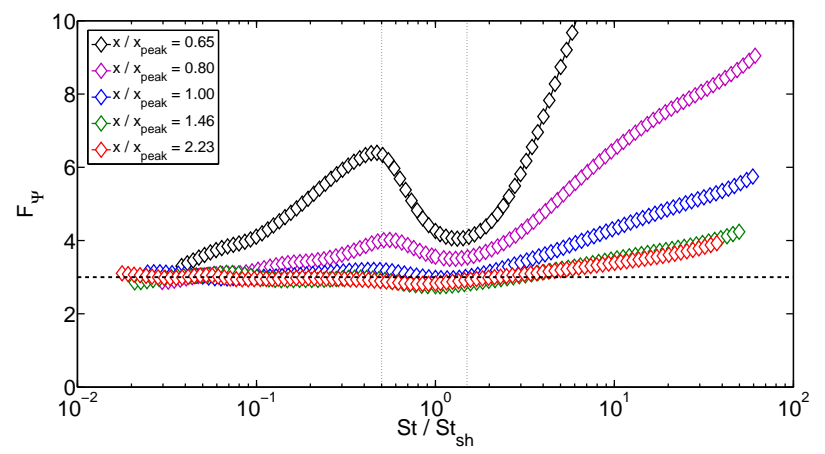

(h) $\mathrm{SSG}+\mathrm{SP} 3$

Figure 18: Skewness (left) and Flatness (right) of the wavelet transforms of $u$ for RG60, FSG17, SSG and SSG+SP3. The vertical dotted lines identify the interval $\Delta S t / S t_{s h}=1$ centred across $S t_{s h}$. The horizontal dashed lines identify $S_{\Psi}=0$ (left) and $F_{\Psi}=3$ (right); $U_{\infty}=17 \mathrm{~m} / \mathrm{s}$.

a bandpass filter to the time-series. The function $\Psi$ can be indeed interpreted as a bandpass filter (Mallat, 1989) whose amplitude depends on the particular choice of $\Psi$. In this analysis $\Psi$ is chosen to be the Mexican 
hat function (second derivative of the Gaussian), as done for example in Nicolleau and Vassilicos (1999) and Mallinson et al. (2004). From on operative point of view, the transforms $u_{\Psi}\left(a, t^{\prime}\right)$ are obtained by making use of the convolution theorem, i.e. the convolution in equation 6 is computed as the inverse Fourier transform of the product of the Fourier transforms of $u$ and $\Psi^{*}$. Next, for every considered value of $a$, we compute the time statistics (third and fourth moments) of the wavelet coefficients.

We consider RG60, FSG17, SSG and SSG+SP3 and, for each grid, we select 5 streamwise positions on the centerline, both in the production region and in the decay region. The locations are chosen in order to make a comparison at similar values of $x / x_{\text {peak }}$ between the different configurations. For each time-series, we limit the wavelet transform analysis to a range of frequencies $\Delta f_{\Psi}=\left[f_{\min }, f_{\max }\right]$. The value of $f_{\min }$ is chosen to be $50 f_{\Theta}$, where $f_{\Theta}$ is the frequency associated with the integral time scale, and $f_{\max }=0.1 f_{\eta}$. The interval $\Delta f_{\Psi}$ is discretised using 100 logarithmically-spaced points.

We examine the skewness (figure 18, left side), $S_{\Psi}=<u_{\Psi}^{3}>/<u_{\Psi}^{2}>^{3 / 2}$, and the flatness (figure 18, right side), $F_{\Psi}=<u_{\Psi}^{4}>/<u_{\Psi}^{2}>^{2}$, of $u_{\Psi}$ as a function of $S t / S t_{s h}$ for the different configurations. We first look at the results for FSG17, since this is the grid where the vortex shedding is the least energetic and persistent. In the production region, $S_{\Psi}$ is negative in a substantial range of $S t$, exhibits a minimum value and is instead near-zero for very small and very large frequencies. When one moves to the decay region, the values of $S_{\Psi}$ become closer to zero or weakly positive, consistent with the trend previously observed for the skewness of $u$ (figure 17b). The FSG17 differs from all the other configurations. In fact, in the production region of RG60, SSG and SSG+SP3 the values of $S_{\Psi}$ undergo a sudden increase towards zero in the proximity of $S t=S t_{s h}$. Analogously, for $S t \approx S t_{s h}$, the values of $F_{\Psi}$ rapidly decrease approaching 3 in the production region, where vortex shedding is strong. On the contrary, when the vortex shedding is weaker, $F_{\Psi}$ generally monotonically increases with increasing frequencies (the small scales are more intermittent), as is always the case for FSG17 (with the exception of the measurements at $x / x_{\text {peak }}=0.62$ ).

Thanks to this analysis, we support and confirm the scenario suggested in section 4.2. In the production region of grid-generated turbulence, there is a correlation between the energy associated with the vortex shedding and the values of the skewness and the flatness of the velocity fluctuations. In particular the skewness and the flatness approach values typical of a Gaussian distribution for an increasing vortex shedding energy contribution. When the vortex shedding is less important, the non-Gaussian behaviour of the velocity fluctuations in the production region is more evident, i.e. the skewness is significantly negative and the flatness is much higher than 3 .

\section{Conclusions}

In this paper we have characterized the flow downstream of different turbulence-generating grids in a wind tunnel, with hot-wire measurements performed mainly on the centerline. In particular we have first considered three types of grids: a regular grid (RG60), a fractal-square grid (FSG17) and a single-square grid (SSG) with the highest value of $t_{0} / L_{0}$.

For FSG17 and SSG, the maximum distance from the grids that we reach is not large enough to capture the classical $C_{\varepsilon}=$ constant behaviour. In fact for both grids the ratio $L_{u} / \lambda$ remains approximately constant in the turbulence decay region, implying $C_{\epsilon} \propto R e_{\lambda}^{-1}$ for a fixed inlet Reynolds number $R e_{t_{0}}$. For RG60, where the wake-interaction length scale $x^{*}$ is the lowest, we do recover the $C_{\varepsilon}=$ constant region of turbulence at $x / x_{\text {peak }} \gtrsim 5.5$.

The Strouhal number $S t_{s h}$ based on $t_{0}$, associated with the vortex shedding from the largest bars of the grids, is the highest for SSG $\left(S t_{s h} \approx 0.19\right)$ and the lowest for FSG17 $\left(S t_{s h} \approx 0.13\right)$. However, when the square root of the area of the bars $\left(\sqrt{t_{0} L_{0}}\right)$ is used for the definition of a Strouhal number $S t_{s h}^{A}$, the values turn out to be approximately the same for both grids, $S t_{s h}^{A} \approx 0.43$ for SSG and $S t_{s h}^{A} \approx 0.44$ for FSG17.

In the production region and close to $x_{\text {peak }}$, the values of turbulence intensity $T u$ are higher for SSG than for FSG17, despite the former having the smallest blockage ratio $\sigma$ and producing the lowest static pressure 
drop $C_{\Delta p}$. This result is achieved by making use of the scaling introduced by Gomes-Fernandes et al. (2012), i.e. increasing the ratio $t_{0} / L_{0}$ for SSG compared to FSG17. However in the turbulence decay region, the values of $T u$ for SSG and FSG17 tend to collapse and they are considerably greater than for RG60, which has the highest $\sigma$ and therefore produces the biggest $C_{\Delta p}$.

In the production region of SSG and even well beyond $x_{\text {peak }}$, a significant contribution to the turbulent kinetic energy $u^{\prime 2}$ comes from the energy associated with a narrow range of $S t$ in the proximity of $S t_{s h}$. This demonstrates that the higher values of $T u$ for SSG are mainly due to vortex shedding effects. We have investigated a novel 3D turbulence generator ( $\mathrm{SSG}+\mathrm{SP}$ ) designed for the study of vortex shedding suppression. We placed a set of four splitter plates in the production region of SSG. Hot-wire measurements were performed along the centerline for 6 distances $x_{s p}$ of the splitter plates from the grid. Among the limited number of positions $x_{s p} / t_{0}$ tested here, the distance $x_{s p} / t_{0}=3$ is found to be the most effective in attenuating the vortex shedding in the present configuration. This value is close to $x_{s p} / D=2.7$, which has been demonstrated to maximise vortex shedding suppression for a single circular cylinder with diameter $D$. For $x_{s p} / t_{0}=3$ the profiles of $T u$ show that (i) the distance $x_{\text {peak }}$ is maximised and (ii) the value of $T u_{\text {peak }}$ is minimised. We deduce that, by attenuating the vortex shedding mechanism, the drag coefficient of the bars $c_{d}$ is reduced, the wakes become narrower and therefore they meet further downstream.

Focusing on the configuration with $x_{s p} / t_{0}=3$ (SSG+SP3), we estimated the energy $E_{s h}$ associated with vortex shedding. In the production region and in the proximity of $x_{p e a k}, E_{s h}$ is reduced for SSG+SP3 with respect to SSG, not only in absolute value but also as a share of $u^{\prime 2}$. For the same $\Delta S t / S t_{s h}$, the ratio $E_{s h} / u^{\prime 2}$ is even lower for the grid FSG17. For FSG17 the vortex shedding effects are less intense and less persistent on the centerline when compared, for the same $x / x_{\text {peak }}$, to all the other tested grids, both with a higher $t_{0}$ (SSG) and with a lower $t_{0}$ (RG60). Hence, similarly to fractal plates (Nedić et al., 2015) and fractal trailing edges (Nedić and Vassilicos, 2015), fractal grids exhibit a weaker vortex shedding. This property could be due to the presence of the smaller geometrical iterations in the fractal geometries. However while for fractal grids the shedding is less persistent downstream, for fractal plates the shedding is more persistent downstream (see Nedić et al., 2015). More research is needed to explain this difference, but for FSG17, the interaction of different wakes with different sizes, which is absent for the other grids investigated here, is suggested to be the cause of the less intense and less persistent vortex shedding along the centreline.

When vortex shedding is suppressed, the growth of the integral length scale $L_{u}$ becomes slower along the centerline. This feature is indeed observed for both SSG+SP3 and for FSG17. In addition to this, in the turbulence decay region the ratio $L_{u} / \lambda$ remains constant even when the splitter plates are added but it is reduced for SSG+SP3 with respect to SSG, despite $R e_{t_{0}}$ being the same.

We have considered the probability density functions (PDFs) of $u$, both in the production and in the decay regions, with particular focus on the evolution of the skewness $S$ and the flatness $F$ of $u$ along the centerline. In the production region the values of $S$ are negative and $F$ is much higher than 3 , in contrast to the decay region where the PDFs are close to a Gaussian distribution. Rare decelerating flow events are therefore more likely to occur than accelerating events in the turbulence production region. These features, previously observed for fractal square grids (Mazellier and Vassilicos, 2010), seem to be intrinsic of the production region, as they are observable for all the turbulence generators here considered. However for FSG17 these characteristics are more pronounced. We have found there is a correlation between the vortex shedding energy in the production region and the values of $S$ and $F$. More specifically when vortex shedding is less prominent, as is the case of FSG17, we find that $S<<0$ and $F>>3$. The non-Gaussian behaviour of $u$ in the production region is "hidden" when vortex shedding becomes important, i.e. $S \rightarrow 0$ and $F \rightarrow 3$ also in the production region in the presence of clear and intense vortex shedding. We checked the consistency of our observations by: (i) comparing $S$ and $F$ for turbulence generators with different $E_{s h}$; (ii) carrying out an exercise (see appendix B) where an artificial shedding contribution is added to a stochastic time-serie; (iii) performing a continuous wavelet transformation of $u$ for all the configurations, both in the production and in the decay region, and comparing the statistics of the wavelet transforms. Further research is still required in order to determine the origin of the strong decelerating events which occur in the turbulence production region. Here we have shown that they are not caused by the 
vortex shedding which in fact masks them in the $S$ and $F$ statistics.

\section{Acknowledgements}

The authors acknowledge support from the EU through the FP7 Marie Curie MULTISOLVE project (Grant Agreement No. 317269). J. C. Vassilicos also acknowledges the support of an ERC Advanced Grant (2013-2018). G. Melina wishes to thank Mr P. Baj for his useful comments during the post-processing of the experimental results. 


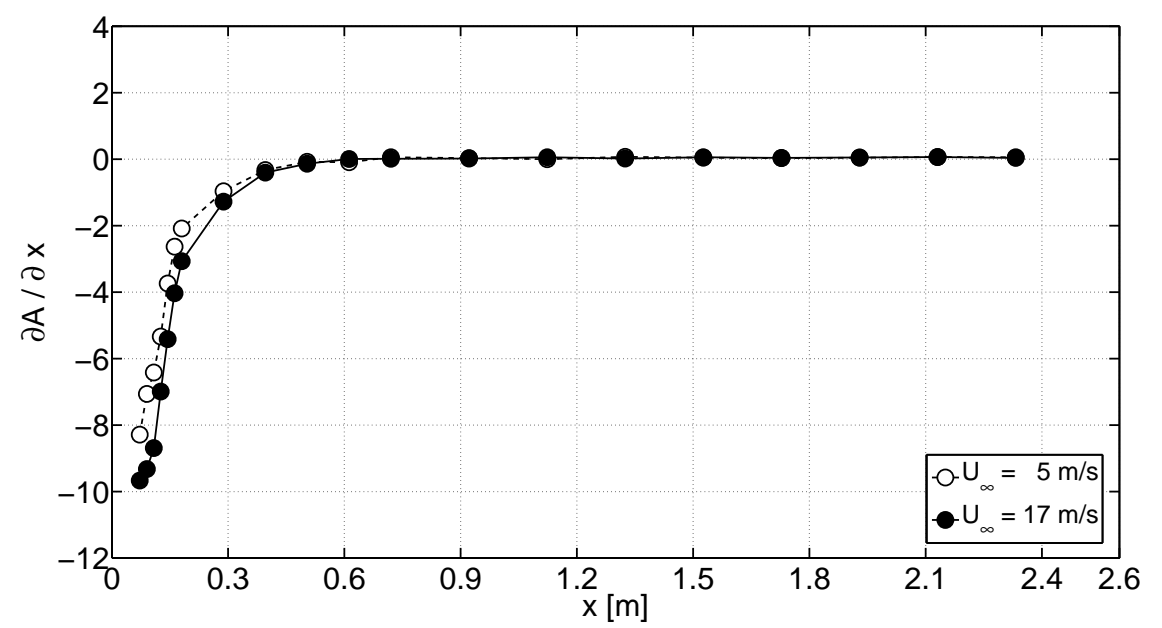

Figure 19: Boundary layer growth for RG60

\section{Appendix A: Growth of the boundary layer on the wind tunnel's walls}

In section 3.1 we have shown that for RG60 the mean velocity increases with $x$ along the centerline for $x>0.6 \mathrm{~m}$ for both $U_{\infty}=5 \mathrm{~m} / \mathrm{s}$ and $U_{\infty}=17 \mathrm{~m} / \mathrm{s}$. In this appendix we show that the increase of $U$ that we have observed is consistent with the growth of a turbulent boundary layer on the wind tunnel's walls.

If we consider the continuity equation it is easy to show that an increase in the boundary layer displacement thickness $\delta^{*}(x)$ leads to an increase in the mean velocity $U(x)$ according to:

$$
\delta^{*}(x) \propto\left(1-\sqrt{\frac{U_{\infty}}{U(x)}}\right)
$$

For a turbulent boundary layer the growth of the displacement thickness can be expressed as (White, 2005):

$$
\frac{\delta^{*}(x)}{x} \propto R e_{x}^{-1 / 7} \propto\left(\frac{U(x) x}{\nu}\right)^{-1 / 7}
$$

From equations A-1 and A-2 we obtain that if $U(x)$ increases as a result of the increase of $\delta^{*}(x)$ it must be verified that:

$$
\left(1-\sqrt{\frac{U_{\infty}}{U(x)}}\right)^{7 / 6}\left(\frac{U(x)}{\nu}\right)^{1 / 6} \propto x
$$

Therefore, letting the left-hand side of equation A-3 to be $A(x)$, it is sufficient to verify that $\partial A / \partial x$ is constant along $x$ in the range where $U(x)$ is found to increase. Figure 19 shows that, for both $U_{\infty}=5 \mathrm{~m} / \mathrm{s}$ and $U_{\infty}=17 \mathrm{~m} / \mathrm{s}, \partial A / \partial x$ is constant along $x$ for $x>0.6 \mathrm{~m}$, which is exactly what we wanted to demonstrate. 


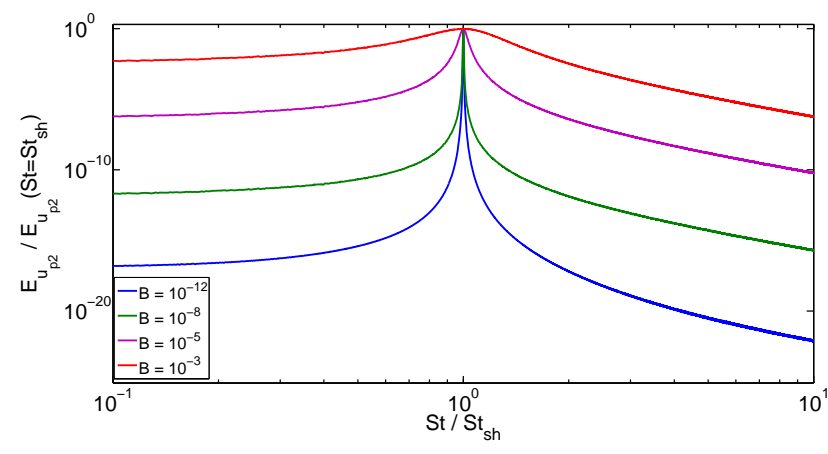

(a)

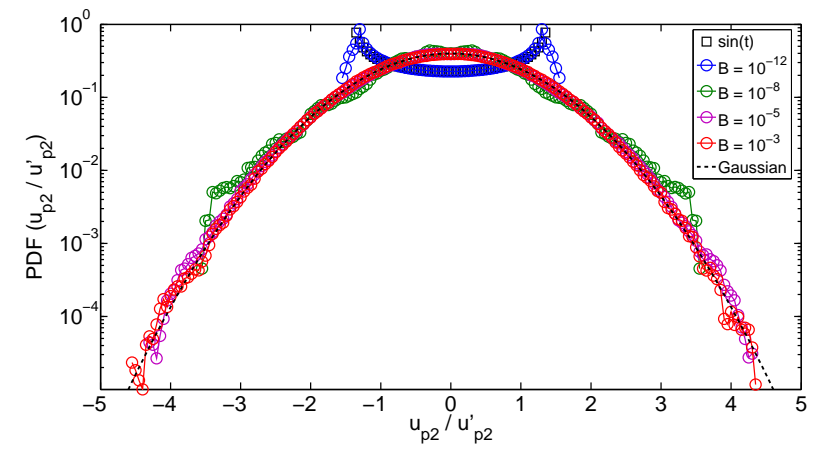

(b)

Figure 20: Power spectrum density (left) and PDF (right) of $u_{p 2}$ for different values of the parameter $B$.

\section{Appendix B: Effect of the vortex shedding energy on the skewness}

In this section we consider a velocity signal resulting from the combination of a periodic component and a stochastic component. We carry out a simple exercise to show that, if the stochastic part is characterized by negative skewness, an increase in the energy associated to the periodic component is consistent with a decrease of the absolute value of the skewness of the total velocity signal.

We consider a stochastic signal $u_{t b 2}(t)$, which is the fluctuating velocity time-serie relative to the hotwire measurement performed for FSG17 at $x / x_{\text {peak }}=0.95$ for $U_{\infty}=17 \mathrm{~m} / \mathrm{s}$. At this location the frequency spectrum does not show any more a local peak at the vortex shedding Strouhal number, $S t_{s h}=0.126$. A periodic component $u_{p 2}(t)$ is modelled with a sinusoidal series given by:

$$
u_{p 2}(t)=\sum_{i} A_{i} \sin \left(2 \pi \frac{S t_{i} U_{\infty}}{t_{0}} t+\phi_{i}\right)
$$

where each $\phi_{i}$ is a random variable with uniform distribution and taking values between $-\pi$ and $\pi$. The coefficients $A_{i}$ are expressed as $A_{i}=\left[B+\left(S t_{i}-S t_{s h}\right)^{2}\right]^{-1}$ in order to distribute most of the energy to the modes associated with $S t$ close to $S t_{s h}$. Increasing the value of the parameter $B$ has the effect of modelling a more brad-band periodic component, as shown by the power spectrum density of $u_{p 2}$ (figure 20a) for different values of $B$. In figure $20 \mathrm{~b}$ we show that with decreasing values of $B$ (modelling a more narrow-band phenomenon) the PDF of $u_{p 2} / u_{p 2}^{\prime}$ tends to the distribution associated with $\sin (t)$, whereas with high values of $B$ the PDF tends to a Gaussian distribution. We now consider a zero-mean signal $u_{t o t}(t)$ given by:

$$
u_{t o t}(t)=u_{p}(t)+u_{t b}(t)
$$

with:

$$
\begin{gathered}
u_{p}(t)=\sqrt{P} \frac{u_{p 2}(t)}{u_{p 2}^{\prime}} \\
u_{t b}(t)=\sqrt{1-P} \frac{u_{t b 2}(t)}{u_{t b 2}^{\prime}}
\end{gathered}
$$

where the parameter $P, 0 \leq P \leq 1$, is the fraction of the total kinetic energy assigned to the periodic component, $P=u_{p}^{\prime 2}$. We always check a posteriori that $u_{t o t}^{\prime 2} \approx 1$, thus verifying that $u_{p}(t)$ and $u_{t b}(t)$ are statistically independent.

At this point we set the parameter $P$ to four different values, $P=0.2,0.4,0.6,0.8$, indicating the increasing importance of the periodic component in the total signal. We compute the skewness of $u_{\text {tot }}(t)$ for the above mentioned values of $P$ and we do this for different choices of $B$. The results are reported in table 3 and they indicate that the skewness decreases (in absolute value) and it approaches zero as the percentage of the energy associated to the periodic component increases, irrespectively of the value of $B$. Figure 21a shows that the 


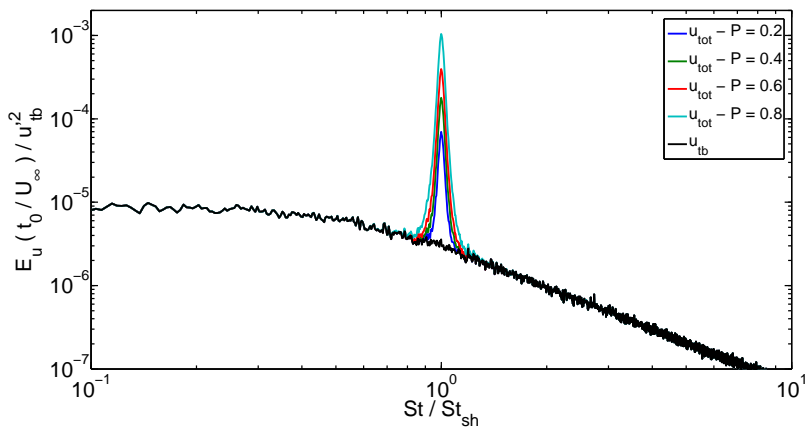

(a)

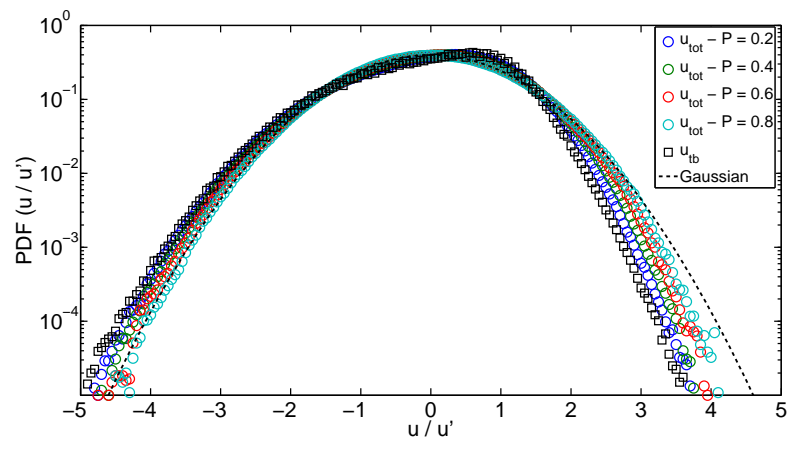

(b)

Figure 21: Power spectrum density (left) and PDF (right) of $u_{t b}$ and $u_{t o t}$ for different values of $P, B=10^{-5}$.

$\mathrm{S}$

\begin{tabular}{ccccc}
$u_{t b}$ & \multicolumn{5}{c}{-0.49} \\
$u_{p}$ & \multicolumn{5}{c}{0.00} & \\
$u_{\text {tot }}(P=0.2)$ & -0.35 & -0.35 & -0.35 & -0.35 \\
$u_{\text {tot }}(P=0.4)$ & -0.23 & -0.22 & -0.23 & -0.22 \\
$u_{\text {tot }}(P=0.6)$ & -0.12 & -0.12 & -0.13 & -0.12 \\
$u_{\text {tot }}(P=0.8)$ & -0.04 & -0.04 & -0.05 & -0.04 \\
& $\left(B=10^{-12}\right)$ & $\left(B=10^{-8}\right)$ & $\left(B=10^{-5}\right)$ & $\left(B=10^{-3}\right)$
\end{tabular}

Table 3: Values of the skewness $S$ of $u_{t b}, u_{p}$ and $u_{t o t}$ for different values of $P$ and $B$.

power spectrum density of $u_{t o t}$ is modified just in the proximity of $S t=S t_{s h}$ by the presence of a more intense periodic contribution, whereas in the remaining part there are non deviations from the case of $u_{t b}$. The effect of $P$ on the PDFs of $u_{t o t}(t) / u_{t o t}^{\prime}$, which become more and more symmetric as $P$ increases, can be seen in figure $21 \mathrm{~b}$ where they are compared to the distribution of $u_{t b}(t) / u_{t b}^{\prime}$ (limit case for $P=0$ ). 


\section{References}

Akilli, H., Sahin, B., and Tumen, N. F. (2005). Suppression of vortex shedding of circular cylinder in shallow water by a splitter plate. Flow Meas. Instrum., 16(4):211-219.

Ali, M. S. M., Doolan, J., and Wheatley, V. (2011). Low Reynolds number flow over a square cylinder with a splitter plate. Phys. Fluids, 23(3):033602.

Anderson, E. A. and Szewczyk, A. A. (1997). Effects of a splitter plate on the near wake of a circular cylinder in 2 and 3-dimensional flow configurations. Exp. Fluids, 23(2):161-174.

Apelt, C. J. and West, G. S. (1975). The effects of wake splitter plates on bluff-body flow in the range $10^{4}<R<5 \times 10^{4}$. Part 2. J. Fluid Mech., 71(1):145-160.

Baj, P., Bruce, P. J. K., and Buxton, O. R. H. (2015). The triple decomposition of a fluctuating velocity field in a multiscale flow. Phys. Fluids, 27:075104.

Cafiero, G., Discetti, S., and Astarita, T. (2015). Flow field topology of submerged jets with fractal generated turbulence. Phys. Fluids, 27:115103.

Cardesa-Dueñas, J. I., Nickels, T. B., and Dawson, J. R. (2012). 2D PIV measurements in the near field of grid turbulence using stitched fields from multiple cameras. Exp. Fluids, 52(6):1611-1627.

Chen, Y. J. and Shao, C. P. (2013). Suppression of vortex shedding from a rectangular cylinder at low Reynolds numbers. J. Fluids Struct., 43:15-27.

Dairay, T., Obligado, M., and Vassilicos, J. C. (2015). Non-equilibrium scaling laws in axisymmetric turbulent wakes. J. Fluid Mech., 781:166-195.

Fail, R., Lawford, J. A., and Eyre, R. C. W. (1959). Low-speed experiments on the wake characteristics of flat plates normal to an airstream. Technical Report 3120, A.R.C. R. \& M.

Farge, M. (1992). Wavelet transforms and their application to turbulence. Annu. Rev. Fluid Mech., 24:395-457.

Goh, K. H. H., Geipel, P., and Lindstedt, R. P. (2014). Lean premixed opposed jet flames in fractal grid generated multiscale turbulence. Combust. Flame, 161(9):2419-2434.

Gomes-Fernandes, R., Ganapathisubramani, B., and Vassilicos, J. C. (2012). Particle image velocimetry study of fractal-generated turbulence. J. Fluid Mech., 711:306-336.

Gomes-Fernandes, R., Ganapathisubramani, B., and Vassilicos, J. C. (2015). The energy cascade in near-field non-homogeneous non-isotropic turbulence. J. Fluid Mech., 771:676-705.

Hurst, D. and Vassilicos, J. C. (2007). Scalings and decay of fractal-generated turbulence. Phys. Fluids, 19(3):035103.

Hwang, J. Y., Yang, K. S., and Sun, S. H. (2003). Reduction of flow-induced forces on a circular ccylinder using a detached splitter plate. Phys. Fluids, 15(8):2433-2436.

Laizet, S., Nedić, J., and Vassilicos, J. C. (2015). The spatial origin of -5/3 spectra in grid-generated turbulence. Phys. Fluids, 27(6):065115.

Laizet, S. and Vassilicos, J. C. (2011). DNS of Fractal-Generated Turbulence. Flow Turbul. Combust., 87(4):673705.

Laizet, S. and Vassilicos, J. C. (2012). Fractal space-scale unfolding mechanism for energy-efficient turbulent mixing. Phys. Rev. E, 86:046302. 
Laizet, S. and Vassilicos, J. C. (2015). Stirring and scalar transfer by grid-generated turbulence in the presence of a mean scalar gradient. J. Fluid Mech., 764:52-75.

Lin, S. Y. and Wu, T. M. (1994). Flow control simulations around a circular cylinder by a finite volume scheme. Numer. Heat Tr. A-Appl., 26(3):301-319.

Mallat, S. G. (1989). A theory for multiresolution signal decomposition: the wavelet representation. IEEE T. Pattern Anal., 2(7):674-693.

Mallinson, S. G., Reizes, J. A., Hong, G., and Westbury, P. S. (2004). Analysis of hot-wire anemometry data obtained in a synthetic jet flow. Exp. Therm. Fluid Sci., 28:265-272.

Maxey, M. R. (1987). The velocity skewness measured in grid turbulence. Phys. Fluids, 30(4):935-938.

Mazellier, N. and Vassilicos, J. C. (2010). Turbulence without Richardson-Kolmogorov cascade. Phys. Fluids, $22(7): 075101$.

Munshi, S. R., Modi, V. J., and Yokomizo, T. (1999). Fluid dynamics of flat plates and rectangular prisms in the presence of moving surface boundary-layer control. J. Wind Eng. Ind. Aerod., 79:37-60.

Nagata, K., Sakai, Y., Inaba, T., Suzuki, H., Terashima, O., and Suzuki, H. (2013). Turbulence structure and turbulence kinetic energy transport in multiscale/fractal-generated turbulence. Phys. Fluids, 25(6):065102.

Nagata, K., Suzuki, H., Sakai, Y., Hayase, T., and Kubo, T. (2008). Direct numerical simulation of turbulent mixing in grid-generated turbulence. Phys. Scripta, T132:014054.

Nedić, J., Ganapathisubramani, B., and Vassilicos, J. C. (2013). Drag and near wake characteristics of flat plates normal to the flow with fractal edge geometries. Fluid Dyn. Res., 45(6):061406.

Nedić, J., Supponen, O., Ganapathisubramani, B., and Vassilicos, J. C. (2015). Geometrical influence on vortex shedding in turbulent axisymmetric wakes. Phys. Fluids, 27(3):035103.

Nedić, J. and Vassilicos, J. C. (2015). Vortex shedding and aerodynamic performance of airfoil with multiscale trailing-edge modifications. AIAA J., 53(11):3240-3250.

Nicolleau, F. and Vassilicos, J. C. (1999). Wavelets for the study of intermittency and its topology. Phil. Trans. R. Soc. Lond. A, 357:2439-2457.

Pope, S. B. (2000). Turbulent Flows. Cambridge University Press, Cambridge.

Roshko, A. (1954). On the drag and shedding frequency of two-dimensional bluff-bodies. Technical Report 3169, NACA.

Seoud, R. E. and Vassilicos, J. C. (2007). Dissipation and decay of fractal-generated turbulence. Phys. Fluids, 19(10):105108.

Suzuki, H., Nagata, K., Sakai, Y., Hayase, T., Hasegawa, Y., and Ushijima, T. (2013). Direct numerical simulation of fractal-generated turbulence. Fluid Dyn. Res., 45(6):061409.

Valente, P. C. and Vassilicos, J. C. (2011). The decay of turbulence generated by a class of multiscale grids. J. Fluid Mech., 687:300-340.

Valente, P. C. and Vassilicos, J. C. (2014). The non-equilibrium region of grid-generated decaying turbulence. J. Fluid Mech., 744:5-37.

Vassilicos, J. C. (2015). Dissipation in Turbulent Flows. Annu. Rev. Fluid Mech., 47:95-114.

Weitemeyer, S., Reinke, N., Peinke, J., and Hölling, M. (2013). Multi-scale generation of turbulence with fractal grids and an active grid. Fluid Dyn. Res., 45(6):061407. 
White, F. M. (2005). Viscous Fluid Flow, $3^{\text {rd }}$ edition, McGraw-Hill, New York.

Zhou, Y., Nagata, K., Sakai, Y., Suzuki, H., Ito, Y., Terashima, O., and Hayase, T. (2014). Relevance of turbulence behind the single square grid to turbulence generated by regular- and multiscale-grids. Phys. Fluids, 26(7):075105. 